\title{
Non-extremal black hole solutions from the $c$-map
}

\author{
D. Errington, ${ }^{a}$ T. Mohaupt ${ }^{a}$ and O. Vaughan ${ }^{b}$ \\ ${ }^{a}$ Department of Mathematical Sciences, University of Liverpool, \\ Peach Street, Liverpool L69 7ZL, U.K. \\ ${ }^{b}$ Department of Mathematics, University of Hamburg, \\ Bundesstraße 55, D-20146 Hamburg, Germany \\ E-mail: david.errington@liv.ac.uk, thomas.mohaupt@liv.ac.uk, \\ owen.vaughan@math.uni-hamburg.de
}

ABSTRACT: We construct new static, spherically symmetric non-extremal black hole solutions of four-dimensional $\mathcal{N}=2$ supergravity, using a systematic technique based on dimensional reduction over time (the $c$-map) and the real formulation of special geometry. For a certain class of models we actually obtain the general solution to the full second order equations of motion, whilst for other classes of models, such as those obtainable by dimensional reduction from five dimensions, heterotic tree-level models, and type-II Calabi-Yau compactifications in the large volume limit a partial set of solutions are found.

When considering specifically non-extremal black hole solutions we find that regularity conditions reduce the number of integration constants by one half. Such solutions satisfy a unique set of first order equations, which we identify.

Several models are investigated in detail, including examples of non-homogeneous spaces such as the quantum deformed STU model. Though we focus on static, spherically symmetric solutions of ungauged supergravity, the method is adaptable to other types of solutions and to gauged supergravity.

KEYwords: Black Holes in String Theory, Black Holes, Supergravity Models

ARXIV EPRINT: 1408.0923 


\section{Contents}

1 Introduction 2

2 Review of vector multiplets and of the $c$-map $\quad 6$

2.1 Four-dimensional vector multiplets $\quad 6$

2.2 Special real coordinates 8

$\begin{array}{lll}2.3 & \text { Dimensional reduction over time } & 10\end{array}$

3 Purely imaginary and spherically symmetric field configurations $\quad 13$

$\begin{array}{lll}3.1 & \text { Purely imaginary field configurations } & 13\end{array}$

$\begin{array}{lll}3.2 & \text { Hessian metrics for PI configurations } & 15\end{array}$

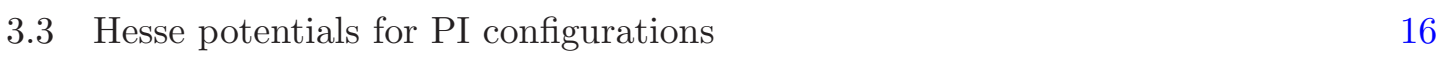

$\begin{array}{lll}3.4 & \text { Spherical symmetry } & 19\end{array}$

4 Three-dimensional instanton solutions $\quad 21$

4.1 Instanton solutions for diagonal models 21

4.2 The universal instanton solution $\quad 24$

4.3 Instanton solutions for block diagonal models 25

$\begin{array}{lll}\text { 4.3.1 The quantum-deformed } S T U \text {-model } & 29\end{array}$

5 Lifting to four dimensions $\quad \mathbf{3 0}$

5.1 General formulas for lifted solutions 31

$\begin{array}{lll}5.2 & \text { Black hole regularity conditions } & 34\end{array}$

5.3 Diagonal models 34

5.3.1 STU-like models $\quad 37$

5.3.2 The $F=i \frac{Y^{1} Y^{2} Y^{3} Y^{4}}{\left(Y^{0}\right)^{2}}$ model $\quad 38$

5.4 Block diagonal models 40

5.4.1 The quantum deformed STU model 41

6 Black holes and first order equations $\quad 42$

$\begin{array}{lll}7 & \text { Conclusion and outlook } & 43\end{array}$

$\begin{array}{ll}\text { A Hessian geometry } & 46\end{array}$

A.1 The Hesse potential $H \quad 46$

$\begin{array}{lll}\text { A.2 The Hesse potential } \tilde{H} & 49\end{array}$

B Spherically symmetric metrics $\quad 51$

$\begin{array}{lll}\text { B.1 Stationary and spherically symmetric } & 51\end{array}$ 


\section{Introduction}

Understanding non-extremal black holes in terms of string theory is the next major step after the earlier discovery of the deep relation between BPS black holes solutions and BPS excitations of strings and branes. Most of the current literature focuses on two ideas: (i) concentrating on models where the scalar manifold is a symmetric space and generating the general solution using group theoretical methods $[1-3] ;^{1}$ (ii) reducing the full second order scalar equations of motion to first order gradient flow equations, thus obtaining a structure similar to BPS solutions [8-17].

In this paper we continue developing a complementary approach which for fivedimensional solutions was developed in [18-21], and for four-dimensional solutions in [22]. We do not assume that the scalar target space is a Riemannian symmetric space, nor that it is homogeneous, but work in the framework of special geometry, which applies to any $\mathcal{N}=2$ supergravity theory and string compactification. Moreover we directly solve the second order field equations and for the subclass of so-called diagonal models (which includes the $S T U$-model, along with other models with non-homogeneous target spaces) we even obtain the most general spherically symmetric solution with purely imaginary scalar fields and half of the gauge charges turned on. We then observe that solutions which correspond to black holes subject to suitable regularity conditions depend only on half of the number of possible integration constants, and satisfy a unique set of first order equations, which we identify. This re-enforces the view that non-extremal solutions preserve some of the features known from BPS solutions.

The fact that certain non-extremal black hole solutions obey unique first order equations has been known for some time in the literature, e.g. [8, 9, 11, 12], but the first order rewriting is imposed as an ansatz and does not exclude the existence of more general nonextremal solutions which cannot obtained this way. In our approach the logic is different: we first find a solution to the full second order equations of motion, and then restrict these solutions to those that correspond to non-extremal black holes. We find that these solutions must satisfy a unique set of first order equations. Moreover, since we observe this feature for a large class of models we expect this to be a common feature for all static, spherically symmetric non-extremal black hole solutions in $\mathcal{N}=2$ supergravity coupled to vector multiplets.

Let us explain the key concepts of our approach, which have been discussed in detail in [22]. Since we are interested in stationary solutions, we perform a dimensional reduction over time and work with the resulting effective three-dimensional Euclidean theory. Threedimensional gravity has no local dynamics, and we can dualise three-dimensional abelian gauge fields into scalars, which leaves us with a non-linear sigma model with some target space $\bar{N}$. Starting with four-dimensional $\mathcal{N}=2$ vector multiplets, where the target space is a projective special Kähler (PSK) manifold $\bar{M}$, the space $\bar{N}$ is a para-quaternionic Kähler manifold [23-25], and the relation between $\bar{M}$ and $\bar{N}$ is the temporal version of

\footnotetext{
${ }^{1}$ Note that in $N=4,8$ supergravity the target manifold is always symmetric. For string theory compactifications with these symmetries the most general BPS and non-extremal black hole solutions have been known for some time [4-7].
} 
the $c$-map. Restricting our attention to spherically symmetric solutions, solving the fourdimensional equations of motion reduces to the problem of finding harmonic maps from the reduced three-dimensional space-'time' to the manifold $\bar{N}$, the image being a geodesic curve parametrised by the three-dimensional scalar fields $[26,27] .{ }^{2}$ We refer to solutions of the three-dimensional Euclidean theory as instantons, although we do not verify explicitly that they have a finite action. Upon lifting these solutions to four dimensions, we find that a subset correspond to regular black hole solutions. Based on the results of $[27,30]$ we expect that after adding a suitable boundary term at least this subset of solutions will have a finite Euclidean action which is related to the ADM mass of the black hole. We refer to extremal (non-extremal) instantons as solutions which lift to extremal (non-extremal) black holes. These solutions correspond to null (non-null) curves in the scalar manifold $\bar{N}$.

We find it useful to use the real formulation of special geometry developed in [22], which is based on a real Hesse potential $H$, rather than the more familiar formulation based on a holomorphic prepotential $F$. The real formulation leads to a more transparent parametrisation of the manifold $\bar{N}$, which in particular allows one to preserve symplectic covariance. In this paper we extend the results of [22] to non-extremal solutions by identifying conditions that lead to an explicit calculation of the Hesse potential and a simplification of the equations of motion. Specifically we will impose that field configurations are spherically symmetric and that the four-dimensional complex scalar fields, typically denoted $z^{A}$, are purely imaginary, or PI, a condition which for models obtainable by reduction from five dimensions is known as axion-free or non-axionic. The PI conditions freeze half of the scalars and eliminate half of the charges. This simplifies the equations of motion to the extent that they take a form similar to the five dimensional case considered before in [18, 19, 21]. Specifically, after imposing spherical symmetry and the PI conditions, the equations of motion can be obtained from variation of the one-dimensional effective Lagrangian

$$
\mathcal{L}=\tilde{H}_{a b}(q)\left(\dot{q}^{a} \dot{q}^{b}-\dot{\hat{q}}^{a} \dot{\hat{q}}^{b}\right)
$$

together with imposing the Hamiltonian constraint. Here the three-dimensional scalar fields $\left(q^{a}, \hat{q}^{a}\right)$ parametrise a pseudo-Riemannian manifold equipped with a Hessian metric $\tilde{H}_{a b}$.

In order to solve the corresponding equations of motion we observe that they decouple into self-contained subsets whenever the scalar metric $\tilde{H}_{a b}$ exhibits a block structure. For each irreducible block we can find at least one independent solution, which contains two free integration constants, in closed form. Thus if the scalar metric $\tilde{H}_{a b}$ decomposes into $m>1$ blocks, we can find a solution which depends on $m$ independent three-dimensional scalar fields. The solution still depends on all $n+1$ charges allowed by the PI conditions, but the ratios between scalar fields belonging to the same block are determined by the ratios of the corresponding gauge charges.

Throughout this paper we will focus on models with prepotentials of the form

$$
F=i^{\lambda-1} \frac{f\left(Y^{1}, \ldots, Y^{n}\right)}{\left(Y^{0}\right)^{\lambda}}, \quad \lambda, n \in \mathbb{Z}^{>0}
$$

\footnotetext{
${ }^{2}$ In gauged supergravity the geodesic equation is modified by a potential, the inclusion of which into the formalism was discussed in [28, 29].
} 
where the holomorphic function $f$ is such that it is real-valued when evaluated on real fields $Y^{I}$. Since $F$ is required to be homogeneous of degree two, $f$ must be homogeneous of degree $\lambda+2$. For this class of models the scalar metric decomposes into at least two blocks, so that we may construct three-dimensional solutions with at least two independent scalar fields, which lift to four-dimensional solutions with at least one non-constant scalar field. While this is not the most general type of solution, it still represents an interesting new type of solution to a large class of models. In particular, models obtainable by dimensional reduction from five dimensions have prepotentials of the form (1.1) with $\lambda=1$ and $f=$ $c_{A B C} Y^{A} Y^{B} Y^{C}$. By means of the M-theory limit and mirror symmetry, any type-II CalabiYau compactification takes this form asymptotically in the large volume/large complex structure limit.

As a concrete example we will consider the quantum deformed $S T U$ model with prepotential $F=-\frac{Y^{1} Y^{2} Y^{3}+a\left(Y^{3}\right)^{3}}{Y^{0}}$. This can be realised, with $a=\frac{1}{3}$, as a heterotic string compactification on $K 3 \times T^{2}$ with instanton numbers $(12,12)$ or $(13,11)$ or $(14,10)$. In this realisation the term proportional to $\left(Y^{3}\right)^{3} / Y^{0}$ arises as a one loop correction [31,32]. Equivalently, the same model can be obtained as a type-IIA compactification on a certain family of elliptically fibred Calabi-Yau three-folds with basis the Hirzebruch surfaces $\mathbb{F}_{0}$ or $\mathbb{F}_{1}$ or $\mathbb{F}_{2}[33]$. In this case all contributions to the prepotential arise at the classical level. We will use this model frequently as an example, as it provides a simple non-homogeneous deformation of the symmetric $S T U$-model, and is one of the simplest examples to study the heterotic/type-II string duality.

Whilst we may generically construct solutions to models of the form (1.1) with two independent scalar fields, more general solutions are possible if the scalar metric decomposes into more than two blocks. One interesting and relevant class are prepotentials which are linear in one field, say $Y^{1} / Y^{0}$, and thus have the structure

$$
F=\frac{f_{1}\left(Y^{1}\right) f_{2}\left(Y^{2}, \ldots Y^{n}\right)}{Y^{0}}
$$

where $f_{1}$ and $f_{2}$ have degree one and two. This class includes all tree-level heterotic prepotentials, which are always linear in the dilaton (see for example [31]), $\mathcal{N}=2$ truncations of $\mathcal{N}=4$ supergravity (see for example [34]), and models based on reducible Jordan algebras (see for example [15]). For prepotentials of the form (1.2) we will show that the scalar metric decomposes into three independent blocks, so that we can obtain three-dimensional non-extremal solutions with three independent scalars.

The limiting case is given by 'diagonal' models, in which the scalar equations of motion decouple completely from one-another and it is possible to obtain the most general stationary solution that satisfies the spherically symmetric and PI conditions. Diagonal models are characterised by prepotentials of the form ${ }^{3}$

$$
F=i^{\lambda-1} \frac{\left(Y^{1} \ldots Y^{n}\right)^{\frac{\lambda+2}{n}}}{\left(Y^{0}\right)^{\lambda}}, \quad \lambda, n \in \mathbb{Z}^{>0} .
$$

\footnotetext{
${ }^{3}$ One can of course obtain equivalent formulations of these models by applying symplectic transformations.
} 
It is known from [35] that homogeneous special Kähler spaces either have prepotentials of the very special form $F=\frac{c_{A B C} Y^{A} Y^{B} Y^{C}}{Y^{0}}$ or are in the $\mathbb{C} H^{n}$ series, where the prepotential is not of diagonal type. It follows that within the diagonal class there are precisely two homogeneous spaces, given by

$$
F=\frac{\left(Y^{1}\right)^{3}}{Y^{0}}, \quad F=\frac{Y^{1} Y^{2} Y^{3}}{Y^{0}},
$$

which correspond to the symmetric spaces $\mathrm{SU}(1,1) / \mathrm{U}(1)$ and $[\mathrm{SU}(1,1) / \mathrm{U}(1)]^{3}$ respectively. All other diagonal models are therefore not homogeneous. As a concrete example of a nonhomogeneous diagonal model we will consider the prepotential $F=i \frac{Y^{1} Y^{2} Y^{3} Y^{4}}{\left(Y^{0}\right)^{2}}$.

We will also discuss one solution that is valid for generic models, i.e. for any choice of holomorphic prepotential $F$, which we will refer to as the universal solution. In this case the three-dimensional scalar fields are all proportional to one-another. In four-dimensions this solutions is characterised by a Reissner-Nordström spacetime metric, $n+1$ electric and magnetic charges, and constant four-dimensional scalar fields $z^{A}$.

The formalism used in this paper has been adapted to gauged supergravity [28, 29] and to the construction of non-extremal rotating solutions [36]. There are similarities between our approach and the H-FGK approach of [37], which builds on [38]. In particular both methods use adapted variables to preserve symmetries and do not rely on group theoretic methods. The H-FGK method was recently used to obtain solutions to type-II models with $\alpha^{\prime}$-corrections [39, 40].

This paper is organised as follows: in section 2 we provide the necessary background on vector multiplets and dimensional reduction (the $c$-map). Key results on Hessian metrics, some of which are not available in the existing literature, are collected in appendix A. In section 3 we analyse field configurations which are spherically symmetric with fourdimensional scalars restricted to purely imaginary values, and derive the resulting simplifications of the three-dimensional equations of motion. Some auxiliary results on spherically symmetric metrics are reviewed in appendix B. In section 4 we solve the three-dimensional equations of motion, while in section 5 we lift these solutions to four dimensions and determine which of these correspond to black holes. While we solve the full second order equations of motion we demonstrate in section 6 that after imposing the regularity conditions required to obtain four-dimensional black holes, our solutions satisfy first order equations. Our conclusions are presented in section 7 .

Throughout this paper $n$ will denote the number of vector multiplets and our index conventions will be:

\begin{tabular}{|l|c|}
\hline Spacetime indices & $\hat{\mu}, \hat{\nu}, \ldots=0,1,2,3$ \\
$\mu, \nu, \ldots=1,2,3$ \\
\hline Target space indices & $A, B, C \ldots=1, \ldots, n$ \\
& $I, J, K, \ldots=0, \ldots, n$ \\
& $a, b, c, \ldots=0, \ldots, 2 n+1$ \\
& $\rho, \sigma, \ldots=1, \ldots, n+1$ \\
& $\alpha, \beta, \gamma, \ldots=0, n+2, \ldots, 2 n+1$ \\
& $\alpha^{\prime}, \beta^{\prime}, \gamma^{\prime}, \ldots=n+2, \ldots, 2 n+1$ \\
\hline
\end{tabular}




\section{Review of vector multiplets and of the c-map}

In this section we review the special geometry of four-dimensional vector multiplets and their dimensional reduction over time (the temporal version of the $c$-map.) This is mostly based on [22], though we also derive new explicit expressions for the inverse Hessian metrics $H^{a b}$ and $\tilde{H}^{a b}$ in $(2.7),(2.17),(2.18)$, which are proved in appendix A.

\subsection{Four-dimensional vector multiplets}

The couplings of four-dimensional $\mathcal{N}=2$ vector multiplets to supergravity were constructed in [41] using the conformal calculus. We refer to [42] for a detailed review. The approach to special geometry taken in [22] is based on the conformal calculus, combined with more recent work in differential geometry, in particular [43, 44].

The bosonic part of the four-dimensional vector multiplet Lagrangian is ${ }^{4}$

$$
\mathrm{e}_{4}^{-1} \mathcal{L}_{4}=\frac{1}{2} R_{4}-g_{A \bar{B}} \partial_{\hat{\mu}} z^{A} \partial^{\hat{\mu}} \bar{z}^{\bar{B}}+\frac{1}{4} F_{\hat{\mu} \hat{\nu}}^{I} \tilde{G}_{I \mid \hat{\mu} \hat{\nu}}
$$

where $R_{4}$ and $\mathrm{e}_{4}$ are the four-dimensional Ricci scalar and vielbein, $\hat{\mu}, \ldots=0, \ldots 3$ are four-dimensional space-time indices, $z^{A}$ with $A=1, \ldots, n$ are complex scalars, $F_{\hat{\mu} \hat{\nu}}^{I}$ with $I=0, \ldots, n$ are abelian field strengths and

$$
G_{I \mid \hat{\mu} \hat{\nu}}:=\mathcal{R}_{I J} F_{\hat{\mu} \hat{\nu}}^{J}-\mathcal{I}_{I J} \tilde{F}_{\hat{\mu} \hat{\nu}}^{J} .
$$

The scalar fields $z^{A}$ parametrise a projective special Kähler (PSK) manifold $\bar{M}$. All couplings in the Lagrangian are encoded in the holomorphic prepotential $\mathcal{F}\left(z^{A}\right)$. The scalar couplings are given by the metric of the PSK manifold $\bar{M}$ :

$$
g_{A \bar{B}}=\partial_{A} \partial_{\bar{B}} K_{\bar{M}}, \quad K_{\bar{M}}=-\log \left(2 i(\mathcal{F}-\overline{\mathcal{F}})-i\left(z^{A}-\bar{z}^{\bar{B}}\right)\left(\mathcal{F}_{A}+\overline{\mathcal{F}}_{\bar{B}}\right)\right),
$$

where we use the notation $\mathcal{F}_{A}=\partial_{A} \mathcal{F}$, etc. The vector field couplings are encoded in the complex matrix $\mathcal{N}_{I J}=\mathcal{R}_{I J}+i \mathcal{I}_{I J}$, which is defined below in (2.4).

The field equations, though not the Lagrangian itself, are invariant under symplectic $\operatorname{Sp}(2 n+2, \mathbb{R})$ transformations, which generalise the electric-magnetic duality of Maxwell theory. This becomes more transparent when using the gauge-equivalent description of the theory in terms of $n+1$ superconformal vector multiplets. Denoting the superconformal scalars as $X^{I}, I=0, \ldots, n$, the couplings of such a theory are encoded in a holomorphic prepotential $F\left(X^{I}\right)$, which is, in addition, homogeneous of degree two: $F\left(\lambda X^{I}\right)=\lambda^{2} F\left(X^{I}\right)$, where $\lambda \in \mathbb{C}^{*}$. The associated scalar metric is

$$
N_{I J}=2 \operatorname{Im} F_{I J}=\frac{\partial K_{M}}{\partial X^{I} \partial \bar{X}^{J}}
$$

with Kähler potential

$$
K_{M}=i\left(X^{I} \bar{F}_{I}-F_{I} \bar{X}^{I}\right) .
$$

\footnotetext{
${ }^{4}$ The sign of the Einstein-Hilbert term is different to [22], due to the fact that in this paper we define the Riemann tensor by $R_{\lambda \mu \nu}^{\rho}=\partial_{\mu} \Gamma_{\nu \lambda}^{\rho}-\partial_{\nu} \Gamma_{\mu \lambda}^{\rho}+\Gamma_{\mu \sigma}^{\rho} \Gamma_{\nu \lambda}^{\sigma}-\Gamma_{\nu \sigma}^{\rho} \Gamma_{\mu \lambda}^{\sigma}$.
} 
Here we use a notation where $F_{I}=\frac{\partial F}{\partial X^{I}}$, etc. The scalars $X^{I}$ parametrise a conical affine special Kähler (CASK) manifold $M$, which is a complex cone over $\bar{M}$. The vector couplings in general involve $R_{I J}=2 \operatorname{Re} F_{I J}$ as well as $N_{I J}$.

Symplectic transformations act by matrices $\mathcal{O}=\left(\mathcal{O}_{b}^{a}\right) \in \operatorname{Sp}(2 n+2, \mathbb{R})$, which are defined by

$$
\mathcal{O}^{T} \Omega \mathcal{O}=\Omega
$$

where

$$
\Omega=\left(\begin{array}{cc}
0 & \mathbb{1} \\
-\mathbb{1} & 0
\end{array}\right)
$$

In the complex formulation of special geometry, the quantities $\left(X^{I}, F_{I}\right)^{T}$ and $\left(F_{\hat{\mu} \hat{\nu}}^{I}, G_{I \mid \hat{\mu} \hat{\nu}}\right)^{T}$ transform as vectors, while $i\left(X^{I} \bar{F}_{I}-F_{I} \bar{X}^{I}\right)$ transforms as a scalar (function). However the holomorphic prepotential $F$ does not transform as a scalar, and $N_{I J}$ and $\mathcal{N}_{I J}$ transform by fractional linear transformations. We will therefore later introduce the real formulation of special geometry where all relevant quantities transform as tensors.

A gauge equivalent formulation of (2.1) is obtained by gauging the superconformal symmetries. After eliminating various auxiliary fields by their equations of motion, the bosonic part of the superconformal Lagrangian is

$$
\mathrm{e}_{4}^{-1} \mathcal{L}_{4}=\frac{1}{2} e^{-\mathcal{K}(X)} R_{4}-e^{-\mathcal{K}(X)} g_{I J} \partial_{\hat{\mu}} X^{I} \partial^{\hat{\mu}} \bar{X}^{J}+\frac{1}{4} e^{-\mathcal{K}(X)} \partial_{\hat{\mu}} \mathcal{K} \partial^{\hat{\mu}} \mathcal{K}+\frac{1}{4} F_{\hat{\mu} \hat{\nu}}^{I} \tilde{G}_{I \mid \hat{\mu} \hat{\nu}},
$$

where

$$
e^{-\mathcal{K}(X)}=-i\left(X^{I} \bar{F}_{I}-F_{I} \bar{X}^{I}\right)
$$

The scalar couplings are

$$
g_{I J}=\frac{\partial^{2} \mathcal{K}}{\partial X^{I} \partial \bar{X}^{J}}=\frac{N_{I J}}{\left(-X^{M} N_{M N} \bar{X}^{N}\right)}+\frac{N_{I K} \bar{X}^{K} N_{J L} X^{L}}{\left(-X^{M} N_{M N} \bar{X}^{N}\right)^{2}}
$$

while the vector couplings are

$$
\mathcal{N}_{I J}=\mathcal{R}_{I J}+i \mathcal{I}_{I J}=\bar{F}_{I J}+i \frac{N_{I K} X^{K} N_{J L} X^{L}}{N_{M N} X^{M} X^{N}}
$$

The Lagrangians (2.2) and (2.1) are gauge equivalent due to the $\mathbb{C}^{*}$-transformations acting on $(2.2)$. The infinitesimal generators of the $\mathbb{C}^{*}$-action are the vector fields

$$
\xi=X^{I} \frac{\partial}{\partial X^{I}}+\bar{X}^{I} \frac{\partial}{\partial \bar{X}^{I}}, \quad J \xi=i X^{I} \frac{\partial}{\partial X^{I}}-i \bar{X}^{I} \frac{\partial}{\partial \bar{X}^{I}},
$$

where $J$ denotes the complex structure of $M$. The resulting finite transformations are

$$
X^{I} \mapsto \lambda X^{I}=|\lambda| e^{i \theta} X^{I}, \quad \lambda \in \mathbb{C}^{*} .
$$

The real scale transformations generated by $\xi$ are homotheties of the CASK metric $N_{I J}$ while the $\mathrm{U}(1)$ transformations generated by $J \xi$ are isometries. 
To recover (2.1) from (2.2) one needs to gauge-fix these transformations. The first step is to impose the D-gauge

$$
e^{-\mathcal{K}(X)}=-i\left(X^{I} \bar{F}_{I}-F_{I} \bar{X}^{I}\right)=1
$$

which fixes the real scale transformations $\mathbb{R}^{>0} \subset \mathbb{C}^{*}$ and brings the Einstein-Hilbert term to its canonical form. The second step is to fix the remaining $U(1) \subset \mathbb{C}^{*}$ transformations. Fixing a U(1) gauge necessarily requires giving up manifest symplectic covariance. Therefore we postpone this step and formulate the c-map in a formalism with manifest symplectic and U(1) covariance. Later, when we construct solutions, the restriction to purely imaginary field configurations will force us to fix a $\mathrm{U}(1)$ gauge, which will be done by imposing $\operatorname{Im} X^{0}=0$. This, and as well any further condition we impose on solutions, restricts symplectic covariance to the subgroup commuting with all conditions.

The gauge equivalence between (2.1) and (2.2) implies that after imposing the Dgauge and taking into account the residual U(1) symmetry the fields $X^{I}$ only represent $2 n$ rather than $2 n+2$ independent real degrees of freedom. This is seen by observing that the tensor $g_{I J}$ is $\mathbb{C}^{*}$-invariant and has a two dimensional kernel $\left(\right.$ since $X^{I} g_{I J}=0=g_{I J} \bar{X}^{J}$ ), which makes modes corresponding to $\mathbb{C}^{*}$-transformations non-propagating. The standard way of obtaining (2.1) from (2.2) is to introduce inhomogeneous special coordinates $z^{A}=$ $X^{A} / X^{0}$, which are $\mathbb{C}^{*}$-invariant, and to verify that (in the D-gauge) $g_{I J} \partial_{\hat{\mu}} X^{I} \partial^{\hat{\mu}} \bar{X}^{J}=$ $g_{A \bar{B}} \partial_{\hat{\mu}} z^{A} \partial^{\hat{\mu}} \bar{z}^{\bar{B}}$. Geometrically, the degenerate tensor $g_{I J}$ is the horizontal lift of the PSK metric $g_{A \bar{B}}$ on $\bar{M}$ to the complex cone $M$. The prepotentials $F\left(X^{I}\right)$ and $\mathcal{F}\left(z^{A}\right)$ are simply related by $\mathcal{F}\left(z^{A}\right)=\left(X^{0}\right)^{-2} F\left(X^{I}\right)=F\left(1, z^{A}\right)$. For the vector kinetic terms one uses that $\mathcal{N}_{I J}$ is homogeneous of degree zero, and therefore $\mathcal{N}_{I J}\left(X^{K}\right)=\mathcal{N}_{I J}\left(1, z^{A}\right)$.

\subsection{Special real coordinates}

We now review and extend the formulation of special Kähler geometry in terms of special real coordinates given in [22]. Special real coordinates were introduced in [43-45], and later used in work on black hole solutions and higher derivative corrections [46-50]. The formalism of [22] provides a formulation of special Kähler geometry in terms of special real coordinates on the CASK manifold associated with the gauge-equivalent superconformal theory. This has the advantage to fully preserve symplectic covariance and can be viewed as an off-shell generalisation of the symplectically covariant formalism used in [51-53] to construct BPS black holes. A different real formalism, which uses special real coordinates on the PSK manifold itself, was developed in [54].

Special real coordinates on the CASK manifold $M$ are defined by

$$
\left(q^{a}\right):=\left(\begin{array}{c}
x^{I} \\
y_{I}
\end{array}\right):=\operatorname{Re}\left(\begin{array}{c}
X^{I} \\
F_{I}
\end{array}\right),
$$

where $a=0, \ldots 2 n+1$. In the real formulation all couplings are encoded in the Hesse potential $H\left(q^{a}\right)$, which is related to the prepotential $F\left(X^{I}\right)$ by a Legendre transformation $\left(x^{I}, u^{I}\right):=\left(\operatorname{Re} X^{I}, \operatorname{Im} X^{I}\right) \rightarrow\left(x^{I}, y_{I}\right):$

$$
H\left(x^{I}, y_{I}\right)=2 \operatorname{Im} F\left(X^{I}(x, y)\right)-2 \operatorname{Im} F_{I}(x, y) y^{I} .
$$


Derivatives of the Hesse potential will be denoted $H_{a}=\frac{\partial H}{\partial q^{a}}$, etc. The Hessian metric

$$
H_{a b}=\frac{\partial^{2} H}{\partial q^{a} \partial q^{b}}
$$

is the real version of the Kähler metric $N_{I J}=2 \operatorname{Im} F_{I J}$ on $M$ in the sense that $N_{I J} d X^{I} d \bar{X}^{J}=$ $H_{a b} d q^{a} d q^{b}$. In special real coordinates, the associated Kähler form is simply

$$
\omega=2 d x^{I} \wedge y_{I}=\Omega_{a b} d q^{a} \wedge d q^{b} .
$$

We will denote the inverse of this matrix by $\Omega^{-1}=\left(\Omega^{a b}\right)$. The special real coordinates are Darboux coordinates. The complex structure takes the form ${ }^{5}$

$$
J_{c}^{a}=-\frac{1}{2} \Omega^{a b} H_{b c}
$$

In special real coordinates the infinitesimal action of $\mathbb{C}^{*}=\mathbb{R}^{>0} \cdot \mathrm{U}(1)$ is generated by the vector fields ${ }^{6}$

$$
\xi=q^{a} \frac{\partial}{\partial q^{a}}, \quad J \xi=\frac{1}{2} H_{a} \Omega^{a b} \frac{\partial}{\partial q^{b}} .
$$

The Hesse potential is homogeneous of degree two under the real scale transformations generated by $\xi$ and invariant under the $\mathrm{U}(1)$ transformations generated by $J \xi$.

Since

$$
e^{-\mathcal{K}(X)}=-i\left(X^{I} \bar{F}_{I}-F_{I} \bar{X}^{I}\right)=-2 H,
$$

the D-gauge corresponds to $-2 H=1$.

The finite transformations generated by $\xi$ and $J \xi$, respectively, are

$$
q^{a} \mapsto|\lambda| q^{a}, \quad q^{a} \mapsto \cos \theta q^{a}+\sin \theta(J q)^{a},
$$

where

$$
(J q)^{a}=J_{b}^{a} q^{b}=-\frac{1}{2} \Omega^{a c} H_{c b} q^{b} .
$$

The Hessian metric $H_{a b}$ can be decomposed as

$$
H_{a b}=(-2 H) H_{a b}^{(0)}+\frac{1}{2 H} H_{a} H_{b}+\frac{2}{H} \Omega_{a c} q^{c} \Omega_{b d} \Omega^{d} .
$$

The tensor $H_{a b}^{(0)}$ is the real version of the degenerate tensor $g_{I J}$,

$$
g_{I J} d X^{I} d \bar{X}^{J}=H_{a b}^{(0)} d q^{a} d q^{b} .
$$

\footnotetext{
${ }^{5}$ This is the standard relation between the complex structure, the Kähler form and the metric of a Kähler manifold. The factor $\frac{1}{2}$ is due to the fact that the matrix representing the Kähler form $\omega$ with respect to the coordinates $q^{a}$ is $2 \Omega_{a b}$.

${ }^{6}$ The special holomorphic coordinates $X^{I}$ and the associated special real coordinates $q^{a}$ are adapted to the $\mathbb{C}^{*}$-action, they are 'conical special coordinates' [27].In terms of such coordinates, which are unique up to linear symplectic transformations, the prepotential and the Hesse potential are homogeneous of degree two with respect to complex and real scale transformations, respectively.
} 
In other words, the scalar term of the bosonic Lagrangian can be rewritten as

$$
e^{-\mathcal{K}(X)} g_{I J} \partial_{\hat{\mu}} X^{I} \partial^{\hat{\mu}} \bar{X}^{J}=(-2 H) H_{a b}^{(0)} \partial_{\hat{\mu}} q^{a} \partial^{\hat{\mu}} q^{b} .
$$

A second, 'dual' set of special real coordinates is given by

$$
q_{a}^{\prime}=H_{a}=\frac{\partial H}{\partial q^{a}}=\left(\begin{array}{c}
2 v_{I} \\
-2 u^{I}
\end{array}\right)=\left(\begin{array}{c}
2 \operatorname{Im} F_{I} \\
-2 \operatorname{Im} X^{I}
\end{array}\right)
$$

where $u^{I}=\operatorname{Im} X^{I}$ and $v_{I}=\operatorname{Im} F_{I}$. Since $H$ is homogeneous of degree two, the special coordinates and dual special coordinates are related by

$$
q_{a}^{\prime}=H_{a b} q^{b} \Leftrightarrow q^{q}=H^{a b} q_{b}^{\prime},
$$

where $H^{a b}$ denotes the inverse of the Hessian metric $H_{a b}$. We thus have two expressions for the CASK metric on $M$ :

$$
g=H_{a b} d q^{a} d q^{b}=H^{a b} d q_{a}^{\prime} d q_{b}^{\prime} .
$$

In appendix A we show that the inverse metric $H^{a b}$ is a Hessian metric with respect to the dual coordinates:

$$
H^{a b}=\frac{\partial^{2} H^{\prime}}{\partial q_{a}^{\prime} \partial q_{b}^{\prime}},
$$

and that the corresponding Hesse potential is $H^{\prime}\left(q^{\prime}\right):=H\left(q\left(q^{\prime}\right)\right)$. For notational simplicity we will often simply write $H\left(q^{\prime}\right)$ instead of the accurate $H^{\prime}\left(q^{\prime}\right){ }^{7}$

Upper indices $a, b, \ldots$ transform with symplectic matrices $\mathcal{O}=\left(\mathcal{O}_{b}^{a}\right)$, while lower indices transform with the contragradient matrices $\mathcal{O}^{T,-1}=:\left(\mathcal{O}_{a}^{b}\right)$. In particular $q^{a}$ transforms as a vector, $q_{a}^{\prime}$ as a co-vector and $H_{a b}$ and $H^{a b}$ as second rank co-tensors and tensors, respectively, while the Hesse potential transforms as a scalar. The raising and lowering of indices with the metric $H_{a b}$ is consistent with symplectic transformations. Moreover, the contraction of tensors with $\Omega_{a b}$ and its inverse is also consistent with symplectic covariance because $\Omega_{a b}$ intertwines between the fundamental and contragradient representation of the symplectic group, i.e. if $q^{a}$ is a symplectic vector then $\Omega_{a b} q^{b}$ is a symplectic co-vector. For example, according to (2.6) the complex structure $J$ acts on $M$ by the diffeomorphism

$$
q^{a} \mapsto(J q)^{a}=-\frac{1}{2} \Omega^{a c} H_{c b} q^{b}=-\frac{1}{2} \Omega^{a c} q_{c}^{\prime}=-\left(\begin{array}{c}
u^{I} \\
v_{I}
\end{array}\right)=-\left(\begin{array}{c}
\operatorname{Im} X^{I} \\
\operatorname{Im} F_{I}
\end{array}\right) .
$$

Thus the vector $(J q)^{a}$ and the co-vector $q_{a}^{\prime}$ are related through multiplication by $\Omega_{a b}$.

\subsection{Dimensional reduction over time}

The four-dimensional space-time metric $g^{(4)}$ and the three-dimensional Euclidean signature metric $g^{(3)}$ are related by

$$
g^{(4)}=-e^{\phi}\left(d t+V_{\mu} d x^{\mu}\right)^{2}+e^{-\phi} g^{(3)},
$$

\footnotetext{
${ }^{7}$ Note that $H(q)$ is in general not invariant under the diffeomorphism $q^{a} \rightarrow q_{a}^{\prime}$, so that is important to interpret $H\left(q^{\prime}\right)$ as $H\left(q\left(q^{\prime}\right)\right)$.
} 
where $\phi$ is the Kaluza-Klein scalar and $V_{\mu}$ the Kaluza-Klein vector. It is useful to combine the Kaluza-Klein scalar with the four-dimensional scalars, which in the superconformal formalism are described by either the holomorphic fields $X^{I}$, or the real fields $q^{a}$, subject to $\mathbb{C}^{*}$-transformations. A key observation is that $\phi$ can be identified with the radial degree of freedom of the cone $M$ over $\bar{M}$, which thus is promoted from a gauge degree of freedom to a physical degree of freedom [22]. In terms of the holomorphic formulation, this is done by defining the rescaled complex symplectic vector

$$
\left(\begin{array}{c}
Y^{I} \\
F_{I}(Y)
\end{array}\right):=e^{\phi / 2}\left(\begin{array}{c}
X^{I} \\
F_{I}(X)
\end{array}\right) .
$$

Here we used that $F_{I}=F_{I}(X)$ is homogeneous of degree one. In the following we will mostly use the rescaled variables $Y^{I}$ and usually denote $F_{I}(Y)$ by $F_{I}$.

If we impose the D-gauge $-i\left(X^{I} \bar{F}_{I}-F_{I} \bar{X}^{I}\right)=1$, this implies that $-i\left(Y^{I} \bar{F}_{I}(Y)-\right.$ $\left.F_{I}(Y) \bar{Y}^{I}\right)=e^{\phi}$, which determines the Kaluza-Klein scalar in terms of the $Y^{I}$. As long as we do not impose a $\mathrm{U}(1)$ gauge the $Y^{I}$ are still subject to $\mathrm{U}(1)$ transformations, but the expression for $e^{\phi}$, and as well the Lagrangian displayed below, are U(1) invariant. The same rescaling can be performed with special real coordinates, or, equivalently, we can modify the definition of special real coordinates by decomposing the complex vector $\left(Y^{I}, F_{I}\right)^{T}$ rather than $\left(X^{I}, F_{I}\right)^{T}$

$$
\begin{gathered}
\left(\begin{array}{c}
x^{I}+i u^{I} \\
y_{I}+i v_{I}
\end{array}\right):=\left(\begin{array}{c}
Y^{I} \\
F_{I}(Y)
\end{array}\right), \\
\left(q^{a}\right):=\left(\begin{array}{c}
x^{I} \\
y_{I}
\end{array}\right):=\operatorname{Re}\left(\begin{array}{c}
Y^{I} \\
F_{I}(Y)
\end{array}\right) .
\end{gathered}
$$

Note that from now on we use special real coordinates which are defined by (2.11) rather than (2.5). Due to the homogeneity of the prepotential all formulas derived using (2.5) are either preserved or modified in a way which is completely determined by the scaling weights of the quantities involved. In the real formalism the Kaluza-Klein scalar is given by

$$
e^{\phi}=-2 H=-i\left(Y^{I} \bar{F}_{I}(Y)-F_{I}(Y) \bar{Y}^{I}\right) .
$$

The three-dimensional theory further contains the scalar fields

$$
\hat{q}^{a}=\left(\frac{1}{2} \zeta^{I}, \frac{1}{2} \tilde{\zeta}_{I}\right)
$$

which descend from the gauge field degrees of freedom. The relation between these threedimensional scalars and the four-dimensional gauge fields can most easily be described via their derivatives

$$
\left(\begin{array}{c}
\partial_{\mu} \zeta^{I} \\
\partial_{\mu} \tilde{\zeta}_{I}
\end{array}\right)=\left(\begin{array}{c}
F_{\mu 0}^{I} \\
G_{I \mid \mu 0}
\end{array}\right) .
$$

While the scalars $\zeta^{I}$ correspond to the time-like components of the four-dimensional vector fields $A_{\mu}^{I}$, the scalars $\tilde{\zeta}_{I}$ are obtained by dualising the reduced, three-dimensional vector 
fields. To obtain a formulation where all propagating bosonic degrees of freedom are scalars, we also dualise the KK-vector $V_{\mu}$ into a scalar field $\tilde{\phi}$ :

$$
\partial_{[\mu} V_{\nu]}=\frac{1}{2 H^{2}} \varepsilon_{\mu \nu \rho}\left(\partial^{\rho} \tilde{\phi}+\frac{1}{2}\left(\zeta^{I} \partial^{\rho} \tilde{\zeta}_{I}-\tilde{\zeta}_{I} \partial^{\rho} \zeta^{I}\right)\right)
$$

In [22] it was shown that the Lagrangian of the three-dimensional theory can be arranged to take the form

$$
\begin{aligned}
\mathrm{e}_{3}^{-1} \mathcal{L}_{3}= & \frac{1}{2} R_{3}-\tilde{H}_{a b}\left(\partial_{\mu} q^{a} \partial^{\mu} q^{b}-\partial_{\mu} \hat{q}^{a} \partial^{\mu} \hat{q}^{b}\right) \\
& -\frac{1}{H^{2}}\left(q^{a} \Omega_{a b} \partial_{\mu} q^{b}\right)^{2}+\frac{2}{H^{2}}\left(q^{a} \Omega_{a b} \partial_{\mu} \hat{q}^{b}\right)^{2} \\
& -\frac{1}{4 H^{2}}\left(\partial_{\mu} \tilde{\phi}+2 \hat{q}^{a} \Omega_{a b} \partial_{\mu} \hat{q}^{b}\right)^{2} .
\end{aligned}
$$

Here $H$ is the Hesse potential, which depends on the rescaled special real coordinates $q^{a}$ and encodes the KK-scalar, and

$$
\tilde{H}_{a b}:=\frac{\partial^{2}}{\partial q^{a} \partial q^{b}} \tilde{H}, \quad \tilde{H}:=-\frac{1}{2} \log (-2 H) .
$$

This tensor can be viewed as a modified metric on $M$, which has been obtained by, essentially, replacing the Hesse potential by its logarithm. We remark that $\tilde{H}_{a b}$ is by construction a symplectic tensor, and that raising and lowering tensor indices using $\tilde{H}_{a b}$ is consistent with symplectic covariance.

We will rely on various properties of the metric $\tilde{H}_{a b}$, which are reviewed or derived in appendix A.2. Here we only mention that it will be convenient later to use dual coordinates with respect to $\tilde{H}_{a b}$ defined by

$$
q_{a}:=\tilde{H}_{a}:=\frac{\partial \tilde{H}}{\partial q^{a}}=\frac{q_{a}^{\prime}}{-2 H},
$$

where $q_{a}^{\prime}=H_{a}$ are the dual coordinates with respect to $H$. Note that since $\tilde{H}_{a}$ is homogeneous of degree -1 :

$$
\tilde{H}_{a b} q^{b}=-\tilde{H}_{a}=-q_{a} \Rightarrow q^{a}=-\tilde{H}^{a b} q_{b} .
$$

One can show that $-\tilde{H}^{\prime}\left(q_{a}\right):=-\tilde{H}\left(q^{b}\left(q_{a}\right)\right)$ is a Hesse potential for the inverse metric $\tilde{H}^{a b}$,

$$
\tilde{H}^{a b}=\frac{\partial q^{a}}{\partial q_{b}}=\frac{\partial^{2}\left(-\tilde{H}^{\prime}\right)}{\partial q_{a} \partial q_{b}} .
$$

In practice we will compute $\tilde{H}^{a b}$ in terms of $H^{\prime \prime}\left(q_{a}\right):=H\left(q^{b}\left(q_{a}\right)\right)$ by

$$
\tilde{H}^{a b}=-\frac{1}{2}\left(\frac{1}{H^{\prime \prime}} \frac{\partial^{2} H^{\prime \prime}}{\partial q_{a} \partial q_{b}}-\frac{1}{H^{2}} \frac{\partial H^{\prime \prime}}{\partial q_{a}} \frac{\partial H^{\prime \prime}}{\partial q_{b}}\right) .
$$

For notational simplicity we will in the following write $H\left(q_{a}\right)$ instead of $H^{\prime \prime}\left(q_{a}\right)=H\left(q^{b}\left(q_{a}\right)\right)$ and $\tilde{H}\left(q_{a}\right)$ instead of $\tilde{H}\left(q^{b}\left(q_{a}\right)\right)$. Note that in general neither $H$ nor $\tilde{H}$ are invariant functions under the diffeomorphism $q^{a} \mapsto q_{a}$. 
The Lagrangian (2.14) is invariant under symplectic transformations and local U(1) transformations. It depends on $4 n+5$ scalars $\left(q^{a}, \hat{q}^{a}, \tilde{\phi}\right)$, but due to the $\mathrm{U}(1)$ gauge symmetry there are only $4 n+4$ independent propagating scalar degrees for freedom. One can gauge fix the $\mathrm{U}(1)$ symmetry by imposing any condition which is transversal to the $\mathrm{U}(1)$ action, and obtain a formulation in terms of $4 n+4$ 'physical' scalar fields. However, such a condition cannot be symplectically invariant and therefore breaks the manifest full symplectic covariance [22]. Finding explicit solutions will require to gauge-fix the U(1) at some point. In our case the gauge-fixing will be implied by a reality condition that we impose on solutions in order to simplify the equations of motion. The solution will still be expressed in terms of symplectic vectors, and manifest invariance under the subgroup of symplectic transformations preserving the reality condition will be preserved. This illustrates that while any transversal condition can be used in principle to fix the $\mathrm{U}(1)$, the type of solution one wants to find typically selects a natural gauge fixing condition. Thus one should not fix a U(1) gauge too early. Geometrically, the $4 n+5$ scalar fields are coordinates on the total space of a $\mathrm{U}(1)$ principal bundle $P$ over the $4 n+4$-dimensional scalar manifold $\bar{N}$ of the three-dimensional theory. Choosing a U(1) gauge allows one to embed $\bar{N}$ into $P$ as a submanifold.

\section{Purely imaginary and spherically symmetric field configurations}

In this section we will analyse the equations of motion given by the variation of (2.14). The full field equations are given in section 6.1 of [22], where a class of four-dimensional stationary solutions were considered. In this paper we will impose two further conditions which greatly simplify the equations of motion, namely that four-dimensional field configurations are

1. purely imaginary,

2. spherically symmetric.

The first is a condition on the target manifold, whilst the second is a condition on spacetime. Let us discuss each condition in turn and investigate the effect they have on the equations of motion.

\subsection{Purely imaginary field configurations}

We will call field configurations purely imaginary if the complex PSK scalars $z^{A}$ are purely imaginary. Since $z^{A}=X^{A} / X^{0}=Y^{A} / Y^{0}$ and we choose the $\mathrm{U}(1)$ gauge fixing condition $\operatorname{Im} Y^{0}=u^{0}=0$ as mentioned in section 2.1, this is equivalent to requiring that $Y^{A}$ are purely imaginary, or in other words

$$
x^{A}=0, A=1, \ldots, n .
$$

For models obtainable by dimensional reduction from five dimensions, the prepotential takes the very special form $F=\frac{c_{A B C} Y^{A} Y^{B} Y^{C}}{Y^{0}}$, with real $c_{A B C}$. In this case the real parts of $z^{A}$ have an axion-like shift symmetry $z^{A} \mapsto z^{A}+\lambda^{A}$, and therefore purely imaginary 
configurations are sometimes referred to as axion-free configurations. In this paper we will be interested in a more general class of models in which the prepotential takes the form

$$
F=i^{\lambda-1} \frac{f\left(Y^{1}, \ldots, Y^{n}\right)}{\left(Y^{0}\right)^{\lambda}},
$$

where $f$ is homogeneous of degree $\lambda+2$ and real when evaluated on real fields. For the particular choice $\lambda=1$ and $f$ a cubic polynomial, this reduces to the class of models obtainable from five dimensions.

For models of the form (3.2) the purely imaginary ('PI') condition (3.1) implies that $F_{0}$ is purely imaginary, or in other words $y_{0}=0$. Denoting by PI the restriction to purely imaginary configurations we have for this class of models

$$
\left.\left(q^{a}\right)_{a=0, \ldots, 2 n+1}\right|_{\mathrm{PI}}=\left(x^{0}, 0, \ldots, 0 ; 0, y_{1}, \ldots, y_{n}\right),
$$

and by acting with the complex structure $J$ one finds

$$
\left.\left(J q^{a}\right)_{a=0, \ldots, 2 n+1}\right|_{\mathrm{PI}}=\left(0, u^{1}, \ldots, u^{n} ; v_{0}, 0, \ldots, 0\right),
$$

so that the PI condition can be expressed in the dual variables as

$$
\left.\left(q_{a}\right)_{a=0, \ldots, 2 n+1}\right|_{\mathrm{PI}}=-\frac{1}{H}\left(v_{0}, 0, \ldots, 0 ; 0,-u^{A}\right) .
$$

Since the PI conditions set half of the entries in certain symplectic vectors to zero, symplectic covariance reduces to the subgroup which preserves this condition. We will see in the following that the equations of motion reduce consistently to a subset of fields, provided that we extend the purely imaginary condition (3.3) to the fields $\hat{q}^{a}$ by

$$
\left.\left(\partial_{\mu} \hat{q}^{a}\right)_{a=0, \ldots, 2 n+1}\right|_{\mathrm{PI}}=\frac{1}{2}\left(\partial_{\mu} \zeta^{0}, 0, \ldots, 0 ; 0, \partial_{\mu} \tilde{\zeta}_{1}, \ldots, \partial_{\mu} \tilde{\zeta}_{n}\right)
$$

Combining expressions (3.3) and (3.6) we find that $q^{a} \Omega_{a b} \partial_{\mu} q^{b}=q^{a} \Omega_{a b} \partial_{\mu} \hat{q}^{b}=0$.

We will later impose spherical symmetry on the four-dimensional solutions, which implies that it is static. In terms of three-dimensional quantities staticity is equivalent to imposing the relation (3.18) given below. For static PI configurations the equations of motion derived from the three-dimensional Lagrangian (2.14), reduce to

$$
\begin{aligned}
\nabla^{\mu}\left[\tilde{H}_{a b} \partial_{\mu} q^{b}\right]-\partial_{a} \tilde{H}_{b c}\left(\partial_{\mu} q^{b} \partial^{\mu} q^{c}-\partial_{\mu} \hat{q}^{b} \partial^{\mu} \hat{q}^{c}\right) & =0 \\
2 \nabla^{\mu}\left[\tilde{H}_{a b} \partial_{\mu} \hat{q}^{b}\right] & =0 \\
\frac{1}{2} R_{\mu \nu}-\tilde{H}_{a b}\left(\partial_{\mu} q^{a} \partial_{\nu} q^{b}-\partial_{\mu} \hat{q}^{a} \partial_{\nu} \hat{q}^{b}\right) & =0
\end{aligned}
$$

with the Kaluza-Klein vector determined by (3.18). These equations of motion follow from the three-dimensional effective Lagrangian

$$
e_{3}^{-1} \mathcal{L}=\frac{1}{2} R_{3}-\tilde{H}_{a b}\left(\partial_{\mu} q^{a} \partial^{\mu} q^{b}-\partial_{\mu} \hat{q}^{a} \partial^{\mu} \hat{q}^{b}\right),
$$

which is obtained by imposing (3.3), (3.6) and (3.18) on (2.14). This shows that the PI conditions represent a consistent truncation. 


\subsection{Hessian metrics for PI configurations}

We now investigate the implications of the PI conditions for the Hessian metric $\tilde{H}_{a b}$. It is convenient to subdivide the range of the index $a=0, \ldots, 2 n+1$ into the ranges $\alpha, \beta, \ldots=$ $0, n+2, \ldots 2 n+1$ and $\rho, \sigma, \ldots=1, \ldots, n+1$. The PI conditions restrict the scalar fields to the PI submanifold defined by

$$
\left(q^{\rho}\right)=\left(x^{A}, y_{0}\right)=0 .
$$

The remaining fields

$$
\left(q^{\alpha}\right)=\left(x^{0}, y_{A}\right)
$$

provide coordinates for the PI submanifold. We will now show that (3.7) and (3.8) correspond to a sigma model which only involves the fields $\left(q^{\alpha}, \hat{q}^{\alpha}\right)$ with couplings determined by the Hessian metric $\tilde{H}_{\alpha \beta}$. The two non-trivial statements we have to prove are: (i) the only surviving terms in the equations of motion involving the first derivatives $\partial_{a} \tilde{H}_{b c}$ of the Hessian metric are of the form $\partial_{\alpha} \tilde{H}_{\beta \gamma}$; (ii) the submatrix $\tilde{H}_{\alpha \beta}$ is a Hessian metric. The rest of this section is devoted to proving these two statements.

From (3.5) we know that the PI conditions can equivalently be written in terms of dual coordinates

$$
\left(q_{\rho}\right)=\left(\tilde{H}_{\rho}\right)=-\frac{1}{H}\left(v_{A},-u^{0}\right)=0,
$$

and the fields

$$
\left(q_{\alpha}\right)=-\frac{1}{H}\left(v_{0},-u^{A}\right)
$$

provide coordinates on the PI submanifold. The splitting of coordinates and dual coordinates into those tangent to the PI submanifold and those transverse to it is consistent with our rules for raising and lowering indices, because the mixed components of the metric vanish on the PI submanifold,

$$
\left.\tilde{H}_{\alpha \rho}\right|_{\mathrm{PI}}=0 .
$$

More generally, since the PI condition $q^{\rho}=0$ implies that $\tilde{H}_{\rho}=0$, it follows that any derivative of $\tilde{H}$ which contains precisely one transverse derivative, vanishes on the PI submanifold $\left.\tilde{H}_{\rho \alpha \beta \cdots}\right|_{\mathrm{PI}}=0[22]$. Next we note that the components $\tilde{H}_{\rho \sigma}$ only appear in the equations of motion contracted with $\partial_{\mu} q^{\rho}$ or $\partial_{\mu} \hat{q}^{\rho}$, which vanish if we impose the PI conditions. Moreover, since $\left.\partial_{\rho} \tilde{H}_{\alpha \beta}\right|_{\mathrm{PI}}=\left.\tilde{H}_{\rho \alpha \beta}\right|_{\mathrm{PI}}=0$, the only surviving terms in the equations of motion involving derivatives of $\tilde{H}_{a b}$ are of the form $\partial_{\gamma} \tilde{H}_{\alpha \beta}$. Together with the vanishing of the mixed components of the Hessian metric, this implies that the only remaining terms in (3.7) are those where $a=\alpha=0, n+2, \ldots, 2 n+1$. We further note that $\tilde{H}_{\alpha \beta}$ is a Hessian metric on the PI submanifold, with Hesse potential $\left.\tilde{H}\right|_{\mathrm{PI}}$ :

$$
\left.\tilde{H}_{\alpha \beta}\right|_{\mathrm{PI}}=\left.\left(\frac{\partial^{2} \tilde{H}}{\partial q^{\alpha} \partial q^{\beta}}\right)\right|_{\mathrm{PI}}=\left(\frac{\left.\partial^{2} \tilde{H}\right|_{\mathrm{PI}}}{\partial q^{\alpha} \partial q^{\beta}}\right),
$$

since this only involves derivatives tangential to the PI submanifold. In the following we will use frequently that whenever a tensor component has one index outside the range $a, b, \ldots=\alpha, \beta, \ldots=0, n+2, \ldots 2 n+1$, it is either zero or decouples from the equations 
of motion. Thus we have shown that the PI conditions amount to a consistent truncation of the three-dimensional Lagrangian to a sigma model for the fields $\left(q^{\alpha}, \hat{q}^{\alpha}\right)$, with scalar metric determined by the Hessian metric $\tilde{H}_{\alpha \beta} \cdot{ }^{8}$

\subsection{Hesse potentials for PI configurations}

It is not possible generically to compute the explicit expression for the Hesse potential corresponding to a prepotential of the form (3.2). This would require solving the relation $\operatorname{Re}\left(F_{I}\left(x^{I}, u^{I}\right)\right)=y_{I}$ to obtain $u^{I}=\operatorname{Im} Y^{I}$ as a function of $\left(x^{I}, y_{I}\right)$, which cannot be done in closed form for a generic prepotential $F$. However, in this section we will show that for any prepotential of the form (3.2) we can find the Hesse potential explicitly as a function of the dual variables $q_{a}$ after restricting to PI field configurations. We will use the following notation for non-vanishing dual variables: $\left(q_{\alpha}\right)=\left(q_{0}, q_{\alpha^{\prime}}\right)$, where $\alpha^{\prime}=n+2, \ldots, 2 n+1$. In terms of these variables, the 'PI Hesse potential' corresponding to (3.2) is

$$
\left.H\left(q_{\alpha}\right)\right|_{\mathrm{PI}}=-\frac{1}{2 \lambda+2}\left[\frac{1}{\lambda^{\lambda}}\left(-q_{0}\right)^{\lambda} f\left(q_{\alpha^{\prime}}\right)\right]^{-\frac{1}{\lambda+1}} .
$$

In the reminder of this section we will derive this formula together with other relations that we will use later to solve the three-dimensional equations of motion and lift the solution to four dimensions.

Starting from (3.2) we compute

$$
F_{0}=-\lambda i^{\lambda-1} \frac{f\left(Y^{1}, \ldots, Y^{n}\right)}{\left(Y^{0}\right)^{\lambda+1}}, \quad F_{A}=i^{\lambda-1} \frac{f_{A}\left(Y^{1}, \ldots, Y^{n}\right)}{\left(Y^{0}\right)^{\lambda}} .
$$

Next we impose the PI condition:

$$
\begin{aligned}
\left.F(x, u)\right|_{\mathrm{PI}} & =i^{\lambda-1} \frac{f\left(i u^{1}, \ldots, i u^{n}\right)}{\left(x^{0}\right)^{\lambda}}, \\
\left.F_{0}\right|_{\mathrm{PI}}=i v_{0} & =-\lambda i^{2 \lambda+1} \frac{f\left(u^{1}, \ldots, u^{n}\right)}{\left(x^{0}\right)^{\lambda+1}},\left.\quad F_{A}\right|_{\mathrm{PI}}=y_{A}=i^{2 \lambda} \frac{f_{A}\left(u^{1}, \ldots, u^{n}\right)}{x^{0^{\lambda}}} .
\end{aligned}
$$

Here we used that $f$ is homogeneous of degree $\lambda+2$ and $f_{A}$ homogeneous of degree $\lambda+1$. Note that since $f$ is by assumption real when evaluated on real fields, $f\left(u^{1}, \ldots, u^{n}\right)$ and $f_{A}\left(u^{1}, \ldots, u^{n}\right)$ are real homogeneous functions. In the following it is understood that $Y^{I}$, $F_{I}$ are subject to the PI condition, and we usually drop the label 'PI'. The relation for $F_{0}$ can be used to solve for $x^{0}$ as a function of the dual coordinates:

$$
\left(x^{0}\right)^{\lambda+1}=(-)^{\lambda+1} \lambda \frac{f(u)}{v_{0}},
$$

where $f(u):=f\left(u^{1}, \ldots, u^{n}\right)$. To obtain $x^{0}$, we need to take the $(\lambda+1)$-st root of the above equation. Since $x^{0}$ must be real, we need to distinguish two cases: between $\lambda+1$ even and $\lambda+1$ odd.

\footnotetext{
${ }^{8}$ The sigma model metric for $\left(q^{\alpha}, \hat{q}^{\alpha}\right)$ is in fact the standard para-Kähler metric on the tangent bundle of the Hessian manifold parametrised by the $q^{\alpha}[18,55]$.
} 
- If $\lambda+1$ is even, then $\left(x^{0}\right)^{\lambda+1}$ is positive so that we must have $f(u) v_{0}>0$. In this case the equation (3.11) has two real roots, corresponding to $x^{0}>0$ and $x^{0}<0$.

- If $\lambda+1$ is odd, $\left(x^{0}\right)^{\lambda+1}$ can be positive or negative, and we obtain no condition on $f(u) v_{0}$ from the reality of $x^{0}$. Moreover the equation (3.11) has a unique real root.

Thus the real solutions of (3.11) are

$$
x^{0}=\phi_{x}\left(\frac{\lambda f(u)}{v_{0}}\right)^{\frac{1}{\lambda+1}},
$$

where

$$
\phi_{x}=\left\{\begin{array}{llll}
\operatorname{sgn}\left(x^{0}\right), & \text { if } \lambda+1 & \text { even }, \\
-1, & \text { if } \lambda+1 & \text { odd },
\end{array}\right.
$$

and where $\operatorname{sgn}\left(x^{0}\right)= \pm 1$ is the sign of $x^{0}$. We now evaluate

$$
e^{-\mathcal{K}(Y)}=-i\left(Y^{I} \bar{F}_{I}-F_{I} \bar{Y}^{I}\right)=-2 H
$$

subject to the PI condition in order to obtain $\left.H(u, v)\right|_{\mathrm{PI}}$ :

$$
\begin{aligned}
e^{-\mathcal{K}(Y)} & =-2 x^{0} v_{0}+2 u^{A} y_{A} \\
& =-2 \phi_{x}\left[\left(\lambda \frac{f(u)}{v_{0}}\right)^{\frac{1}{\lambda+1}} v_{0}+(\lambda+2) f(u)\left(\frac{v_{0}^{\lambda}}{\lambda^{\lambda} f(u)^{\lambda}}\right)^{\frac{1}{\lambda+1}}\right],
\end{aligned}
$$

where we used that $\phi_{x}^{\lambda}=(-1)^{\lambda+1} \phi_{x}$ and $\phi_{x}=\phi_{x}^{-1}$ and $u^{A} f_{A}=(\lambda+2) f(u)$. Next we move the linear factors $v_{0}$ and $f(u)$ inside the roots. If $\lambda+1$ is even we need to split off a factor -1 if $v_{0}$ is negative:

$$
v_{0}=\operatorname{sgn}\left(v_{0}\right)\left(v_{0}^{\lambda+1}\right)^{\frac{1}{\lambda+1}},
$$

whereas for odd $\lambda+1$ there is no such factor. Let us therefore define

$$
\phi_{v}=\left\{\begin{array}{llll}
\operatorname{sgn}\left(v_{0}\right), & \text { if } \lambda+1 & \text { even }, \\
1, & \text { if } \lambda+1 & \text { odd } .
\end{array}\right.
$$

We also need an analogous sign factor $\phi_{f}$ for $f$, but it turns out that $\phi_{f}=\phi_{v}$. This is clear because for $\lambda+1$ odd we know that $\phi_{f}=1$, whereas for $\lambda+1$ even we know that $f$ and $v_{0}$ have the same sign. We can thus combine terms to obtain

$$
e^{-\mathcal{K}(Y)}=-\phi_{x} \phi_{v}(4 \lambda+4)\left(\frac{f(u) v_{0}^{\lambda}}{\lambda^{\lambda}}\right)^{\frac{1}{\lambda+1}} .
$$

Since $e^{-\mathcal{K}(Y)}$ must be positive we obtain constraints on the signs of $f, v_{0}$ and $x^{0}$. If $\lambda+1$ is even, the root is only real when $f v_{0}^{\lambda}>0$, which is not a new condition as it is already implied by the reality of $x^{0}$. Positivity of $e^{-\mathcal{K}(Y)}$ requires $\phi_{x} \phi_{v}<0$, which implies that $x^{0}$ and $v_{0}$ (and hence $f$ ) have opposite sign. If $\lambda+1$ is odd, then $-\phi_{x} \phi_{v}=1$ holds automatically, and we obtain $f>0$ as the only condition. 
The conditions on $f, v_{0}$ and $x^{0}$ can be summarised as follows

- If $\lambda+1$ is even, then either $f(u)>0, v_{0}>0$ or $f(u)<0, v_{0}<0$. Moreover the sign of $x^{0}$ must be opposite to that of $v_{0}$, which enters into the solution through $\phi_{x}=\operatorname{sgn}\left(x^{0}\right)$.

- If $\lambda+1$ is odd, then $f(u)>0$, and $\phi_{x}=-1$.

Equivalently:

$f(u)$ and $v_{0}$ must satisfy

$$
v_{0}^{\lambda} f(u)>0,
$$

and if $\lambda+1$ is even the signs of $v_{0}$ and $x^{0}$ must be opposite.

For later use we note that the Hesse potential, restricted to configurations satisfying the PI condition, is

$$
\left.H(u, v)\right|_{\mathrm{PI}}=-(2 \lambda+2)\left[\frac{1}{\lambda^{\lambda}} v_{0}^{\lambda} f\left(u^{1}, \ldots, u^{n}\right)\right]^{\frac{1}{\lambda+1}},
$$

and the non-zero dual scalars are given by

$$
q_{0}=-\frac{v_{0}}{H(u, v)}, \quad q_{\alpha^{\prime}}=q_{A+(n+1)}=\frac{u^{A}}{H(u, v)} .
$$

Using that $f(u)$ is homogeneous of degree $\lambda+2$, we can rewrite (3.14) in terms of $q_{a}$ :

$$
\left.H\left(u\left(q_{\alpha}\right), v\left(q_{\alpha}\right)\right)\right|_{\mathrm{PI}}=-\frac{1}{2 \lambda+2}\left[\frac{1}{\lambda^{\lambda}}\left(-q_{0}\right)^{\lambda} f\left(q_{\alpha^{\prime}}\right)\right]^{-\frac{1}{\lambda+1}} .
$$

For notational convenience we will set $H\left(q_{\alpha}\right):=\left.H\left(u\left(q_{\alpha}\right), v\left(q_{\alpha}\right)\right)\right|_{\mathrm{PI}}$ in the following. ${ }^{9}$

Let us explain how to check that this expression is real and negative, as required. Similar arguments can be used as quick checks for the correctness of the various explicit solutions we give later. All we need to do is to re-write the conditions (3.13) in terms of the dual variables $q_{a}$. First note that since $H<0$ and $q_{0}=-H^{-1} v_{0}$, it follows that $q_{0}$ and $v_{0}$ have the same sign. Next, $q_{A+(n+1)}=H^{-1} u^{A}$, so that $q_{A+(n+1)}$ and $u^{A}$ have opposite signs. Since $f(u)=H^{-(\lambda+2)} f\left(q_{\alpha^{\prime}}\right)$, where $\alpha^{\prime}=A+(n+1)$, it follows that $f\left(q_{\alpha^{\prime}}\right)$ has the same (opposite) sign to $f(u)$ if $\lambda+1$ is odd (even). Thus the conditions for consistent real solutions are:

$q_{0}$ and $f\left(q_{\alpha^{\prime}}\right)$ must satisfy

$$
\left(-q_{0}\right)^{\lambda} f\left(q_{\alpha^{\prime}}\right)>0
$$

If $\lambda+1$ is even, then the sign of $x^{0}$ must be opposite to that of $q_{0}$, which enters into solutions through $\phi_{x}=\operatorname{sgn}\left(x^{0}\right)$. If $\lambda+1$ is odd, then $\phi_{x}=-1$.

From this criterion it is manifest that $H\left(q_{a}\right)$ as given in (3.15) is real and negative.

Note that even for purely imaginary field configurations it is still not possible to find an explicit expression for the Hesse potential in terms of $\left(x^{I}, y_{I}\right)$ without imposing further conditions. One class where this is possible are the diagonal models, which will be discussed in section (4.1).

\footnotetext{
${ }^{9}$ In the notation of section A.2 the correct notation would be $H^{\prime \prime}\left(q_{a}\right)$. Note that $H\left(q_{a}\right)$ is not an invariant function under the diffeomorphism $\left(u^{I}, v_{I}\right) \mapsto q_{a}$.
} 


\subsection{Spherical symmetry}

Besides the PI condition we impose that all four-dimensional fields (metric, scalars and gauge fields) are spherically symmetric. Spherically symmetry spacetime metrics are reviewed in appendix B.1. According to (B.2) the three-dimensional part of any fourdimensional stationary and spherically symmetric spacetime metric can be written in the form

$$
g^{(3)}=e^{4 \mathcal{A}(\tau)} d \tau^{2}+e^{2 \mathcal{A}(\tau)} d \Omega_{(2)}^{2} .
$$

In terms of the radial coordinate $\tau$ the three-dimensional Laplacian takes the simple form $\Delta=\frac{d^{2}}{d \tau^{2}}+\cdots$, where the omitted terms are independent of $\tau$. The solution for the scalar fields corresponds to a geodesic curve in the scalar manifold $\bar{N}$ of the three-dimensional theory. The advantage of the radial coordinate $\tau$ compared to other (not affinely related) choices of a radial coordinate is that $\tau$ provides an affine parametrisation of this geodesic.

It turns out that the three-dimensional Einstein equations completely fix the function $\mathcal{A}(\tau)$. Discarding solutions that are periodic in $\tau$ one finds that $e^{-\mathcal{A}(\tau)}=\frac{\sinh c \tau}{c}$, for some constant $c$. The three-dimensional metric then takes the form [26]

$$
g^{(3)}=\frac{c^{4}}{\sinh ^{4} c \tau} d \tau^{2}+\frac{c^{2}}{\sinh ^{2} c \tau} d \Omega_{(2)}^{2},
$$

which is precisely the three-dimensional part of the Reissner-Nordström metric.

For the interpretation as a dimensionally reduced black hole, it is convenient to replace the 'affine' radial coordinate $\tau$, by a different radial coordinate $\rho$, defined by the relation

$$
W(\rho):=1-\frac{2 c}{\rho}=e^{-2 c \tau},
$$

in which case

$$
g^{(3)}=\frac{d \rho^{2}}{W}+\rho^{2} d \Omega_{(2)}^{2} .
$$

The parameter $c \geq 0$ is the non-extremality parameter, with $c=0$ being the extremal limit. The outer horizon is at $\rho=2 c$, which corresponds to $\tau \rightarrow \infty$. Using the radial coordinate $\rho$, the solution can be continued analytically from the outer horizon to the inner horizon located at $\rho=0$.

Combining the fact that spacetime is both spherically symmetric and stationary is enough to ensure that is is static, the proof of which is reviewed in appendix B.1. Therefore one may choose coordinates in which the KK-vector vanishes in (2.8). In terms of threedimensional fields this means that

$$
\frac{1}{2 H}\left(\partial_{\mu} \tilde{\phi}+\frac{1}{2}\left(\zeta^{I} \partial_{\mu} \tilde{\zeta}_{I}-\tilde{\zeta}_{I} \partial_{\mu} \zeta^{I}\right)\right)=\frac{1}{2 H}\left(\partial_{\mu} \tilde{\phi}+2 \hat{q}^{c} \Omega_{c d} \partial_{\mu} \hat{q}^{d}\right)=0
$$

This term (squared) appears in isolation in the Lagrangian (2.14) and therefore decouples from all other terms in the equations of motion. An effective Lagrangian for general static configurations is given by taking the first two lines of (2.14). If we impose in addition the PI conditions, then this reduces to the first line, with half the scalar fields being constant, as discussed previously in this section. If we impose spherical symmetry together with 
the PI conditions, then staticity is implied, and all fields can be taken to only depend on the affine radial coordinate $\tau$ using the parametrisation (3.16). Then the equations (3.7) reduce to

$$
\begin{aligned}
\frac{d}{d \tau}\left(\tilde{H}_{a b} \dot{q}^{b}\right)-\partial_{a} \tilde{H}_{b c}\left(\dot{q}^{b} \dot{q}^{c}-\dot{\hat{q}}^{b} \dot{\hat{q}}^{c}\right) & =0, \\
\frac{d}{d \tau}\left(\tilde{H}_{a b} \dot{\hat{q}}^{b}\right) & =0, \\
\tilde{H}_{a b}\left(\dot{q}^{b} \dot{q}^{c}-\dot{\hat{q}}^{b} \dot{\hat{q}}^{c}\right) & =c^{2},
\end{aligned}
$$

where a dot denotes the differentiation with respect to $\tau$. The first two equations are the scalar equations of motion, which are equivalent to the geodesic equation for the curve $\left(q^{a}(\tau), \hat{q}^{a}(\tau)\right)$ on the scalar manifold $\bar{N}$. The scalar equations of motion follow from the one-dimensional effective Lagrangian

$$
\mathcal{L}_{1}=-\tilde{H}_{a b}\left(\dot{q}^{a} \dot{q}^{b}-\dot{\hat{q}}^{a} \dot{\hat{q}}^{b}\right)
$$

The third equation of (3.19), which is the non-trivial component of the higher-dimensional Einstein equations, is the Hamiltonian constraint which needs to be imposed on top of the one-dimensional Euler-Lagrange equations.

In our applications it will be convenient to use the inverse metric $\tilde{H}^{a b}$ and the dual coordinates $q_{a}$. To perform the rewriting we use the relations (A.4) and (A.5), and the relation $\partial_{d} \tilde{H}^{a b}=-\tilde{H}^{a c} \tilde{H}^{b e} \partial_{d} \tilde{H}_{c e}$ between the first derivatives of a metric and those of its inverse. Note that indices on the vector fields $\dot{q}^{a}, \dot{\hat{q}}^{a}$ and derivatives $\partial_{a}=\frac{\partial}{\partial q^{a}}$ are raised and lowered with $\tilde{H}_{a b}$, in particular that $\tilde{H}^{a b} \frac{\partial}{\partial q^{b}}=\frac{\partial}{\partial q_{a}}$. In terms of the dual variables, the scalar equations of motion are

$$
\ddot{q}_{a}+\frac{1}{2} \tilde{H}_{a d} \partial^{d} \tilde{H}^{b c}\left(\dot{q}_{a} \dot{q}_{b}-\dot{\hat{q}}_{a} \dot{\hat{q}}_{b}\right)=0,
$$

and

$$
\ddot{\hat{q}}_{a}=0,
$$

and the Hamiltonian constraint takes the form

$$
\tilde{H}^{b c}\left(\dot{q}_{a} \dot{q}_{b}-\dot{\hat{q}}_{a} \dot{\hat{q}}_{b}\right)=c^{2},
$$

where $\dot{\hat{q}}_{a}:=\tilde{H}_{a b} \dot{\hat{q}}^{b}$ is the co-vector field obtained by lowering the index of the vector field $\dot{\hat{q}}^{a}$.

We remark that we do not require the existence of 'dual coordinates' $\hat{q}_{a}$ as functions on the scalar manifolds. In particular it is not possible to define dual coordinates as $\tilde{H}_{a b} \hat{q}^{b}$ (unless $\tilde{H}_{a b}$ is constant), because this would not be consistent with $\dot{\hat{q}}_{a}=\tilde{H}_{a b} \dot{\hat{q}}^{b}$. However $\hat{q}^{a}$ are well defined functions on the scalar manifold, and $\dot{\hat{q}}^{a}$ and $\dot{\hat{q}}_{a}$ are well defined (co-)vector fields.

The remainder of this paper is dedicated to solving the equations of motion written in the dual coordinates (3.21)-(3.23). It is worth reiterating that we have only imposed that solutions are stationary, spherically symmetric and purely imaginary. Recall that the latter condition means that

$$
q_{\rho}=\dot{\hat{q}}_{\rho}=0, \quad \rho=1, \ldots, n+1,
$$


and, as discussed in 3.2, this implies that the equations of motion (3.21)-(3.23) only involve the fields

$$
\left(q_{\alpha}, \dot{\hat{q}}_{\alpha}\right), \quad \alpha=0, n+2, \ldots 2 n+1 .
$$

With this in mind, the $\dot{\hat{q}}_{a}$ equation of motion can be immediately integrated to give

$$
\dot{\hat{q}}_{a}=K_{a}=\left(-\mathcal{Q}_{0}, 0, \ldots, 0 ; 0, \mathcal{P}^{1}, \ldots, \mathcal{P}^{n}\right),
$$

where the integration constants $\mathcal{Q}_{0}, \mathcal{P}^{A}$ are proportional to the electric and magnetic charges of the black hole solution. ${ }^{10}$ The $n+1$ charges $\mathcal{Q}_{0}, \mathcal{P}^{A}$ are the maximum number allowed for purely imaginary configurations, and they may be freely chosen for all solutions considered in this paper, regardless of the model in question.

\section{Three-dimensional instanton solutions}

We will now construct explicit solutions to the equations (3.21)-(3.23), which we refer to as instanton solutions.

\subsection{Instanton solutions for diagonal models}

We start by discussing a class of models where we will be able to find the general purely imaginary solution in closed form. The prepotential is restricted to have the form

$$
F=i^{\lambda-1} \frac{\left(Y^{1} \ldots Y^{n}\right)^{\frac{\lambda+2}{n}}}{\left(Y^{0}\right)^{\lambda}} .
$$

For reasons that will become clear we refer to this class as diagonal models. They form a two-parameter family parametrised by $\lambda, n=1,2,3, \ldots$. The particular choice $\lambda=1, n=3$ corresponds to the well-known $S T U$ model. We will see that the family of diagonal models shares certain features of the $S T U$-model, in particular they allow for explicit solutions, although such models do in general not correspond to homogeneous spaces.

According to (3.9) after imposing the PI conditions we can write the Hesse potential for this class of models as

$$
H\left(q_{a}\right)=-\frac{1}{2 \lambda+2}\left[\left(\frac{-q_{0}}{\lambda}\right)^{\lambda}\left(q_{n+2} \ldots q_{2 n+1}\right)^{\frac{\lambda+2}{n}}\right]^{-\frac{1}{\lambda+1}} .
$$

This is manifestly real and negative for $\left(-q_{0}\right)^{\lambda}\left(q_{n+2} \ldots q_{2 n+1}\right)^{\frac{\lambda+2}{n}}>0$, which is $(3.13)$ expressed in terms of $q_{a}$. Both the $q_{a}$ equation of motion (3.21) and the Hamiltonian constraint (3.23) require us to compute the matrix $\tilde{H}^{a b}$ given by

$$
\tilde{H}^{a b}=\frac{1}{2 H} \frac{\partial^{2} H}{\partial q_{a} \partial q_{b}}-\frac{1}{2 H^{2}} \frac{\partial H}{\partial q_{a}} \frac{\partial H}{\partial q_{b}}, \quad H=H\left(q_{a}\right),
$$

which follows from (A.8) by setting $C=-\frac{1}{2}$.

\footnotetext{
${ }^{10}$ The minus sign in front of $\mathcal{Q}_{0}$ is included in view of the relation $q_{a}=\frac{1}{H}\left(-v_{I}, u^{I}\right)$. Our sign conventions are such that for BPS solutions the attractor equations take the same form as in [53].
} 
Before entering into explicit calculations, we can already observe that for prepotentials of the from (4.1) the Hessian metric for PI field configurations exhibits further simplifications compared to the general class (3.2). By taking the logarithm of (4.2) we obtain

$$
\tilde{H} \sim \log \left(q_{0}\right)^{-\frac{\lambda}{\lambda+1}}+\log \left(q_{n+2}\right)^{-\frac{\lambda+2}{(\lambda+1) n}}+\cdots+\log \left(q_{2 n+1}\right)^{-\frac{\lambda+2}{(\lambda+1) n}},
$$

from which it is easy to see that applying (4.3) leads to a matrix with the following block structure

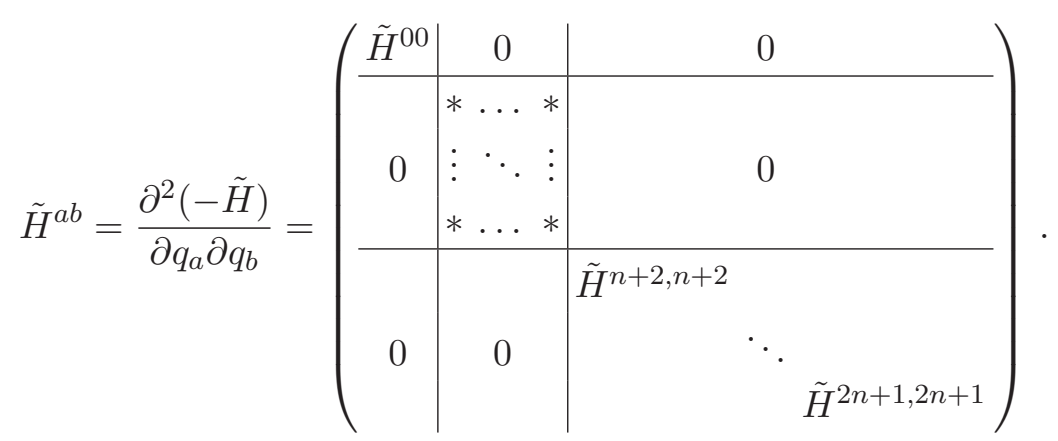

The central $(n+1) \times(n+1)$ block $\tilde{H}^{\rho \sigma}$ contains unknown and potentially nonzero entries that we represent with a ' $*$.' However, we have shown that for PI field configurations this block decouples from the equations of motion. We also observe the vanishing of mixed entries of the form $\tilde{H}^{\alpha \rho}$, as derived previously in generality. The additional simplification, which is obvious from the fact that $\tilde{H}$ given in (4.4) is a sum of terms each depending on precisely one coordinate, is that the submatrix $\tilde{H}^{\alpha \beta}$ is diagonal. This phenomenon, which motivates the terminology 'diagonal models' was already observed in [18] for fivedimensional extremal black holes, and [22] for four-dimensional extremal black holes and in [21] for extremal and non-extremal black strings.

Using (4.3), we find the nonzero entries relevant for the equations of motion, and their derivatives, to be

$$
\begin{aligned}
\tilde{H}^{00} & =\frac{\lambda}{2 \lambda+2} q_{0}^{-2}, & \partial^{0} \tilde{H}^{00} & =-2 \frac{\lambda}{2 \lambda+2} q_{0}^{-3} \\
\tilde{H}^{n+2, n+2} & =\frac{\lambda+2}{(2 \lambda+2) n} q_{n+2}^{-2}, & \partial^{n+2} \tilde{H}^{n+2, n+2} & =-2 \frac{\lambda+2}{(2 \lambda+2) n} q_{n+2}^{-3}, \\
& \vdots & & \\
\tilde{H}^{2 n+1,2 n+1} & =\frac{\lambda+2}{(2 \lambda+2) n} q_{2 n+1}^{-2}, & \partial^{2 n+1} \tilde{H}^{2 n+1,2 n+1} & =-2 \frac{\lambda+2}{(2 \lambda+2) n} q_{2 n+1}^{-3} .
\end{aligned}
$$

Note that each diagonal matrix element only depends on the corresponding scalar field, thus leading to a complete decoupling of the scalar equations of motion. Because of the diagonal structure of $\tilde{H}^{a b}$, the inverse elements, $\tilde{H}_{a b}$, of the above entries are easy to obtain e.g.

$$
\tilde{H}_{00}=\left(\tilde{H}^{00}\right)^{-1}=\frac{1}{\tilde{H}^{00}}=\frac{2 \lambda+2}{\lambda} q_{0}^{2}
$$


and similarly for the other components. We can use this to compute the quantities $\frac{1}{2} \partial_{a} \tilde{H}^{b c}=\frac{1}{2} \tilde{H}_{a d} \partial^{d} \tilde{H}^{b c}$ that appear in the equations of motion

$$
\begin{aligned}
\frac{1}{2} \partial_{0} \tilde{H}^{00} & =-q_{0}^{-1} \\
\frac{1}{2} \partial_{n+1+A} \tilde{H}^{n+1+A, n+1+A} & =-q_{n+1+A}^{-1},
\end{aligned}
$$

where $A=1, \ldots, n$.

Replacing $\dot{\hat{q}}_{a}$ by $K_{a}$ according to (3.24), the $q_{a}$ equation of motion (3.21) becomes

$$
\ddot{q}_{a}+\frac{1}{2} \tilde{H}_{a d} \partial^{d} \tilde{H}^{b c}\left(\dot{q}_{b} \dot{q}_{c}-K_{b} K_{c}\right)=0 .
$$

Substituting from (4.9) and (3.24), we see that the individual equations look like

$$
\begin{array}{crlrl}
a & =0, & \ddot{q}_{0}-q_{0}^{-1}\left(\dot{q}_{0}^{2}-\mathcal{Q}_{0}^{2}\right) & =0, \\
a & =n+2, & \ddot{q}_{n+2}-q_{n+2}^{-1}\left(\dot{q}_{n+2}^{2}-\left(\mathcal{P}^{1}\right)^{2}\right) & =0, \\
\vdots & & \\
a & =2 n+1, & \ddot{q}_{2 n+1}-q_{2 n+1}^{-1}\left(\dot{q}_{2 n+1}-\left(\mathcal{P}^{n}\right)^{2}\right) & =0 .
\end{array}
$$

These equations are solved by

$$
\begin{aligned}
q_{0} & = \pm \frac{-\mathcal{Q}_{0}}{B_{0}} \sinh \left(B_{0} \tau+B_{0} \frac{h_{0}}{\mathcal{Q}_{0}}\right), \\
q_{n+2} & = \pm \frac{\mathcal{P}^{1}}{B^{1}} \sinh \left(B^{1} \tau+B^{1} \frac{h^{1}}{\mathcal{P}^{1}}\right), \\
\vdots & \\
q_{2 n+1} & = \pm \frac{\mathcal{P}^{n}}{B^{n}} \sinh \left(B^{n} \tau+B^{n} \frac{h^{n}}{\mathcal{P}^{n}}\right),
\end{aligned}
$$

where $B_{0}, B^{A}, h_{0}, h^{A}$ are integration constants. Since making the replacement $B_{0}, B^{A} \mapsto$ $-B_{0},-B^{A}$ leaves the solution invariant we may assume without loss of generality that the integration constants $B_{0}, B^{A}$ are non-negative. The choice of sign distributions in (4.13) has an interesting effect when lifting to four-dimensional black holes: when taking the extremal limit one obtains BPS black holes for the case where all signs are equal, whereas for all other sign distributions one obtains non-BPS black holes. We will not address this further in the present paper, but refer the reader to [22] for more information on this topic. For convenience we will choose the positive sign in the above expressions from now on.

Having eliminated $\dot{\hat{q}}_{a}$ by their equation of motion the Hamiltonian constraint (3.23) becomes a condition on the the scalar fields $q_{a}$. We can use (4.6)-(4.7) and (3.24) to expand this as

$$
\begin{aligned}
\frac{\lambda}{2 \lambda+2} q_{0}^{-2}\left(\dot{q}_{0}^{2}-K_{0}^{2}\right)+\frac{\lambda+2}{(2 \lambda+2) n} q_{n+2}^{-2}\left(\dot{q}_{n+2}^{2}-K_{n+2}^{2}\right) \\
+\cdots+\frac{\lambda+2}{(2 \lambda+2) n} q_{2 n+1}^{-2}\left(\dot{q}_{2 n+1}^{2}-K_{2 n+1}^{2}\right)=c^{2} .
\end{aligned}
$$


Substituting our solution for the scalars $q_{a}$ into this, we see the Hamiltonian constraint becomes

$$
\frac{\lambda}{2 \lambda+2}\left(B_{0}\right)^{2}+\frac{\lambda}{(2 \lambda+2) n}\left(B^{1}\right)^{2}+\cdots+\frac{\lambda}{(2 \lambda+2) n}\left(B^{n}\right)^{2}=c^{2},
$$

which can be viewed either as a constraint on the integration constants $B_{0}, B^{A}$ or on the non-extremality parameter, $c$.

The instanton solution for $q_{a}$ and $\dot{\hat{q}}_{a}$ given in (4.13) and (3.24) respectively, subject to the Hamiltonian constraint (4.15), is general in the sense that for $2(n+1)$ independent scalar fields $q_{\alpha}, \hat{q}_{\alpha}, \alpha=0, n+2, \ldots 2 n+1$ subject to second order field equations we have $4(n+1)$ integration constants. These may be counted as follows: if we regard $c$ as a dependent quantity, then $2(n+1)$ integration constants are given by $B_{0}, B^{A}, h_{0}, h^{A}$ appearing in the solution of the $q_{a}$ equation of motion. The charges $\mathcal{Q}_{0}, \mathcal{P}^{A}$ provide a further $n+1$ integration constants. The remaining $n+1$ integration constants, which are obtained by integrating $\dot{\hat{q}}_{a}=K_{a}$ are unphysical due to the axionic shift symmetries of the fields $\hat{q}^{a}$, which reflect the four-dimensional gauge symmetry.

We remark that while we have not defined dual coordinates $\hat{q}_{a}$ as functions on the scalar manifold, one can of course integrate $\dot{\hat{q}}_{a}=K_{a}$ along the curve representing the solution, and thus obtain functions $\hat{q}_{a}(\tau)=K_{a} \tau+R_{a}$ along that curve. Alternatively, $\hat{q}^{a}$ are well defined functions on the scalar manifold, an integration of $\dot{\hat{q}}^{a}=\tilde{H}^{a b} K_{b}$ will involve $n+1$ integration constants. However these integration constants will drop out of any four-dimensional gauge invariant quantity, so that only $3(n+1)$ integration constants are relevant. We will see later that four-dimensional black hole regularity conditions reduce this further to $2(n+1)$ integration constants, which reflects the existence of a unique first order rewriting of the $q^{a}$ equations of motion.

We further remark that using the explicit expressions (4.6)-(4.7) we can obtain an explicit expression for the Hesse potential $\left.H(x, y)\right|_{\mathrm{PI}}$ in terms of the special real variables $q^{a}=\left(x^{I}, y_{I}\right)$, restricted to PI configurations:

$$
\left.H\left(q^{a}\right)\right|_{\mathrm{PI}}=C\left[\left(-q^{0}\right)^{\lambda}\left(q^{n+2} \cdots q^{2 n+1}\right)^{\frac{\lambda+2}{n}}\right]^{\frac{1}{\lambda+1}},
$$

where $C$ is a numerical constant that does not enter into the expression $\tilde{H}_{a b}$. Note that by expressing $q_{a}$ in terms of $q^{a}$ the power $-\frac{1}{\lambda+1}$ gets replaced by its negative.

\subsection{The universal instanton solution}

The opposite case to a diagonal model is a model where $\tilde{H}^{a b}$, after imposing the PI conditions, does not admit a further block decomposition, so that every non-vanishing scalar field $q_{\alpha}$ couples with all others. In this case we can still find a solution with one independent three-dimensional scalar field by taking the fields $q_{\alpha}$ to be proportional to each other

$$
q_{\alpha}=\xi_{\alpha} q,
$$

where the constants $\xi_{\alpha}$ will turn out to be determined by the charges. For $q$ we take the same solution as for scalars in diagonal models,

$$
q= \pm \frac{K}{B} \sinh \left(B \tau+\frac{B h}{K}\right)
$$


Thus $q$ satisfies

$$
\ddot{q}=\frac{\dot{q}^{2}-K^{2}}{q}=B^{2} q \Rightarrow \frac{\dot{q}^{2}-K^{2}}{q^{2}}=B^{2} .
$$

Note that $\ddot{q}_{\alpha}=B^{2} q_{\alpha}$. The homogeneity properties of the Hessian metric imply [19]

$$
q_{\alpha}=-\tilde{H}_{\alpha \beta} q^{\beta}=\frac{1}{2} q^{\gamma} \partial_{\gamma} \tilde{H}_{\alpha \beta} q^{\beta}=\frac{1}{2} \partial_{\alpha} \tilde{H}_{\beta \gamma} q^{\beta} q^{\gamma}=-\frac{1}{2} \partial_{\alpha} \tilde{H}^{\beta \gamma} q_{\beta} q_{\gamma}=-\frac{1}{2} \tilde{H}_{\alpha \delta} \partial^{\delta} \tilde{H}^{\beta \gamma} q_{\beta} q_{\gamma} .
$$

Using this when substituting back (4.16) into the $q_{a}$ equation of motion we obtain

$$
\tilde{H}_{\alpha \delta} \partial^{\delta} \tilde{H}^{\beta \gamma}\left(K^{2} \xi_{\beta} \xi_{\gamma}-K_{\beta} K_{\gamma}\right)=0 .
$$

This can be solved by imposing the constraint

$$
\xi_{\alpha}=\frac{K_{\alpha}}{K}
$$

which fixes the constants of proportionality between the scalars $q_{\alpha}$ in terms of the charges $K_{\alpha}$, up to the overall scale $K$, which drops out of ratios:

$$
\frac{\xi_{\alpha}}{\xi_{\beta}}=\frac{q_{\alpha}}{q_{\beta}}=\frac{K_{\alpha}}{K_{\beta}} .
$$

It remains to solve the Hamiltonian constraint. Here we use that $\tilde{H}^{\alpha \beta}$ is homogeneous of degree -2 in the variables $q_{\alpha}$ :

$$
\tilde{H}^{\alpha \beta}\left(q_{\alpha}\right)=q^{-2} \tilde{H}^{\alpha \beta}\left(\xi_{\alpha}\right) .
$$

Then the Hamiltonian constraint becomes

$$
\frac{\tilde{H}^{\alpha \beta}(\xi)}{q^{2}}\left(\xi_{\alpha} \xi_{\beta} \dot{q}^{2}-K_{\alpha} K_{\beta}\right)=B^{2} \tilde{H}^{\alpha \beta}(\xi) \xi_{\alpha} \xi_{\beta}=c^{2} .
$$

This is an algebraic constraint which fixes $B$ in terms of $c$ and the charges. We will see in section 5.3 that the universal solution corresponds to a four-dimensional solution with the non-extremal Reissner-Nordström metric, multiple charges, and constant four-dimensional scalars.

\subsection{Instanton solutions for block diagonal models}

In this section we explain how to obtain explicit instanton solutions for non-diagonal models, assuming that $\tilde{H}^{\alpha \beta}$ decomposes into two or more blocks. We will show that in this case we can obtain explicit solutions which still carry all the gauge charges consistent with the PI conditions, but with a reduced number of independent scalar fields, because the solutions for scalar fields belonging to the same block will be proportional, with ratios determined by the ratios of the corresponding gauge charges.

To keep formulas simple we will only consider the case $\lambda=1$, with prepotentials of the form

$$
F=\frac{f\left(Y^{1}, \ldots, Y^{n}\right)}{Y^{0}}
$$


with $f\left(Y^{1}, \ldots, Y^{n}\right)$ homogeneous of degree 3 , and real when evaluated on real fields. The corresponding Hesse potential for PI configurations is

$$
H\left(q_{0}, q_{\alpha^{\prime}}\right)=-\frac{1}{4}\left[\left(-q_{0}\right) f\left(q_{\alpha^{\prime}}\right)\right]^{-\frac{1}{2}} .
$$

Note that all 'very special' prepotentials that can be obtained by dimensional reduction are of this type. As observed in [22], when imposing the PI conditions it follows that $\tilde{H}^{0 \beta^{\prime}}=0$ for $\beta^{\prime}=n+2, \ldots, 2 n+1$, so that $\tilde{H}^{\alpha \beta}$ always subdivides into at least two blocks, $\tilde{H}^{00}$ and a further $n \times n$ block $\tilde{H}^{\alpha^{\prime} \beta^{\prime}}$ :

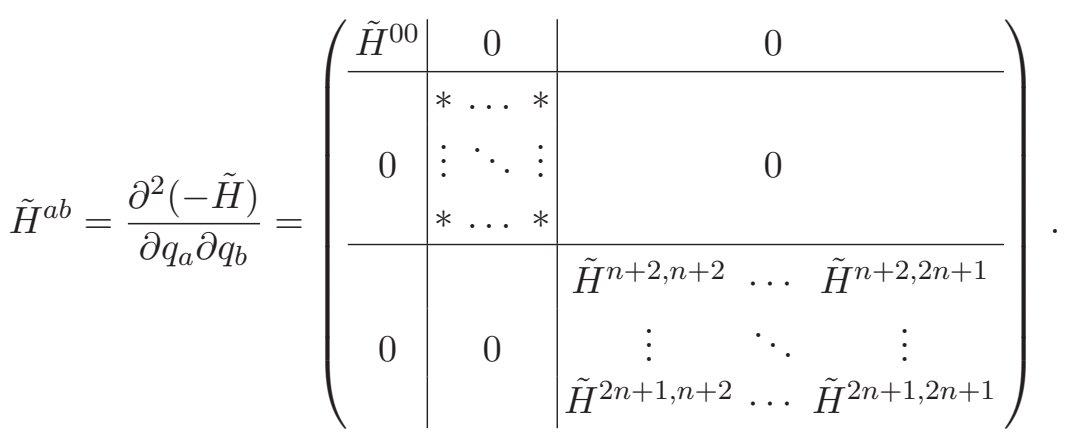

If one restricts the form of $f\left(Y^{1}, \ldots, Y^{n}\right)$ then $\tilde{H}^{\alpha^{\prime} \beta^{\prime}}$ might decompose into further blocks, ${ }^{11}$ the limiting case being diagonal models.

To be precise, a block decomposition of the equations of motion does not only require that $\tilde{H}^{\alpha \beta}$ exhibits a block structure. The full set of conditions is obtained in the same way as when we discussed the consistent truncation of the equations of motion by the PI condition in section 3. To have a decoupling one also needs that the matrix elements in each block only depend on the scalar fields corresponding to this block. This implies in particular that the derivatives $\partial^{\gamma} \tilde{H}^{\alpha \beta}$ exhibit the same block decomposition as $\tilde{H}^{\alpha \beta}$ itself. For terminological convenience we will refer to these conditions as $\tilde{H}^{\alpha \beta}$ 'admitting a block decomposition.' The conditions are met for (4.17), and all the further examples that we will discuss. It is clear that a block decomposition always occurs if $H$ is a product with factors depending on disjoint subsets of variables, so that $\tilde{H}$ is a sum of terms depending on disjoint subsets of variables, which implies that $\tilde{H}^{\alpha \beta}$ is a product metric. ${ }^{12}$

One important class of examples which always allows a further block decomposition are prepotentials of the form

$$
F=\frac{f_{1}\left(Y^{1}\right) f_{2}\left(Y^{2}, \ldots, Y^{n}\right)}{Y^{0}} .
$$

This class contains tree-level heterotic prepotentials, which are always linear in the dilaton $Y^{1} / Y^{0}, \mathcal{N}=2$ truncations of $\mathcal{N}=4$ theories, and models based on reducible Jordan algebras. The corresponding Hesse potential for PI configurations is

$$
H\left(q_{0}, q_{n+2}, q_{n+3}, \ldots\right)=-\frac{1}{4}\left[\left(-q_{0}\right) f_{1}\left(q_{n+2}\right) f_{2}\left(q_{n+3}, q_{n+4}, \ldots\right)\right]^{-\frac{1}{2}},
$$

\footnotetext{
${ }^{11}$ One might of course need to perform row operations to make the decomposition explicit.

${ }^{12}$ Further examples can arise whenever the further consistent truncation of a model induces a decoupling of the field equations for the remaining fields. We will not investigate this systematically in the present paper.
} 
so that

$$
\tilde{H}^{a b}=\left(\begin{array}{c|ccc|cccc}
\frac{1}{4} q_{0}^{-2} & 0 & \cdots & 0 & 0 & 0 & 0 & \cdots \\
\hline 0 & * & \cdots & * & 0 & 0 & 0 & \cdots \\
\vdots & \vdots & & \vdots & 0 & 0 & 0 & \cdots \\
0 & * & \cdots & * & 0 & 0 & 0 & \cdots \\
\hline 0 & 0 & 0 & 0 & * & 0 & 0 & \cdots \\
0 & 0 & 0 & 0 & 0 & * & * & \cdots \\
0 & 0 & 0 & 0 & 0 & * & * & \cdots \\
\vdots & \vdots & \vdots & \vdots & \vdots & \vdots & \vdots & \ddots
\end{array}\right) .
$$

Whenever a model is not diagonal its scalar fields will couple, which will prevent us from finding the general solution by the method used in the previous section. However, if $\tilde{H}^{\alpha \beta}$ has a block decomposition, then only the scalars corresponding to the same block couple to one another. One can then proceed by taking all scalar fields belonging to the same block to be proportional. In this case the method described in section 4.2 gives a nontrivial solution, though not the most general one since one only has as many independent scalar fields as one has blocks.

Let us assume that there are $M$ blocks, labeled by $m=1, \ldots, M$. For each block we take all the corresponding scalars to be proportional to

$$
q_{(m)}= \pm \frac{K^{(m)}}{B^{(m)}} \sinh \left(B^{(m)} \tau+\frac{B^{(m)} h^{(m)}}{K^{(m)}}\right) .
$$

Since the blocks decouple this solves the $q_{a}$ equations of motion for the $m$-th block, with constants of proportionality fixed by the corresponding charges:

$$
\xi_{a}^{(m)}=\frac{K_{a}^{(m)}}{K^{(m)}}, \quad \frac{\xi_{a}^{(m)}}{\xi_{b}^{(m)}}=\frac{q_{a}^{(m)}}{q_{b}^{(m)}}=\frac{K_{a}^{(m)}}{K_{b}^{(m)}},
$$

where the indices $a, b, \ldots$ are restricted to values corresponding to the $m$-th block. In the following we will omit the superscript $(m)$ on $\xi_{a}$ and $K_{a}$ whenever it is clear to which block they belong.

The Hamiltonian constraint (3.23) couples the scalars in different blocks:

$$
\sum_{m=1}^{M} \sum_{a, b \in I_{(m)}} \tilde{H}^{a b}(\xi) \frac{\xi_{a} \xi_{b} \dot{q}_{(m)}^{2}-K_{a} K_{b}}{q_{(m)}^{2}}=\sum_{m=1}^{M}\left(B^{(m)}\right)^{2} \psi_{m}=c^{2},
$$

where

$$
\psi_{m}=\sum_{a, b \in I_{(m)}} \tilde{H}^{a b}(\xi) \xi_{a} \xi_{b},
$$

with $I_{(m)}$ the subset of indices corresponding to the $m$-th block. We remark that we will see in section 5.4 that for regular black hole solutions $B^{(m)}=c$ for all $m$, so that the condition

$$
\sum_{m=1}^{M} \psi_{m}=1
$$

must be satisfied. 
For prepotentials of the form (3.2), subject to the PI conditions, there will always be a single $1 \times 1$ block corresponding to the field $q_{0}$. A decomposition of the complementary block $q_{n+2}, \ldots q_{2 n+1}$ will occur for special choices of the function $f\left(Y^{1}, \ldots, Y^{n}\right)$, as for the example given by (4.19), (4.20). For illustration, consider the case

$$
H\left(q_{0}, q_{\alpha^{\prime}}\right)=-\frac{1}{4}\left[\left(-q_{0}\right) f_{1}\left(q_{(n+1)+1}, \ldots, q_{(n+1)+k}\right) f_{2}\left(q_{(n+1)+(k+1)}, \ldots q_{2 n+1}\right)\right]^{-\frac{1}{2}},
$$

where the bottom-right entries $\tilde{H}^{\alpha^{\prime} \beta^{\prime}}$ split into two sub-blocks of size $k \times k$ and $l \times l$ where $k \geq 1$ and $l=n-k$. In this case there are three independent scalar fields which we can take to be $q_{0}, q_{(1)}=q_{n+2}, q_{(2)}=q_{(n+1)+(k+1)}$. Using the parameters $\xi_{a}$ we can express all charges in terms of three 'independent charges', namely $\mathcal{Q}_{0}$ and

$$
\begin{aligned}
& \mathcal{P}^{(1)}:=\mathcal{P}^{1}=\frac{1}{\xi_{n+3}} \mathcal{P}^{2}=\ldots=\frac{1}{\xi_{(n+1)+k}} \mathcal{P}^{k}, \\
& \mathcal{P}^{(2)}:=\mathcal{P}^{k+1}=\frac{1}{\xi_{n+2+k+1}} \mathcal{P}^{k+2}=\ldots=\frac{1}{\xi_{2 n+1}} \mathcal{P}^{n},
\end{aligned}
$$

where we used that we have chosen $\xi_{n+2}=\xi_{(n+1)+(k+1)}=1$. Note that the solution still depends on all $n+1$ charges $\mathcal{Q}_{0}, \mathcal{P}^{A}$, which can be chosen freely, but then determine the ratios between scalar fields belonging to the same block. It is however convenient to express block-diagonal solutions in terms of charges $\mathcal{Q}_{0}, \mathcal{P}^{(1)}, \mathcal{P}^{(2)}$ which are in one-to-one correspondence with the independent scalar fields $q_{0}, q_{(1)}, q_{(2)}$. As we will check below, this system of independent fields and corresponding charges can be interpreted as a consistent truncation of the full system.

After eliminating the fields $\dot{\hat{q}}_{a}$ by their equations of motion the field equations for the independent scalar fields are

$$
\begin{array}{r}
\ddot{q}_{0}-\frac{\left[\dot{q}_{0}^{2}-\mathcal{Q}_{0}^{2}\right]}{q_{0}}=0, \\
\ddot{q}_{(1)}-\frac{\left[\dot{q}_{(1)}^{2}-\mathcal{P}^{(1) 2}\right]}{q_{(1)}}=0, \\
\ddot{q}_{(2)}-\frac{\left[\dot{q}_{(2)}^{2}-\mathcal{P}^{(2) 2}\right]}{q_{(2)}}=0,
\end{array}
$$

which are solved by

$$
\begin{aligned}
q_{0} & = \pm \frac{-\mathcal{Q}_{0}}{B_{0}} \sinh \left(B_{0} \tau+B_{0} \frac{h_{0}}{\mathcal{Q}_{0}}\right) \\
q_{(1)} & = \pm \frac{\mathcal{P}^{(1)}}{B^{(1)}} \sinh \left(B^{(1)} \tau+B^{(1)} \frac{h^{(1)}}{\mathcal{P}^{(1)}}\right) \\
q_{(2)} & = \pm \frac{\mathcal{P}^{(2)}}{B^{(2)}} \sinh \left(B^{(2)} \tau+B^{(2)} \frac{h^{(2)}}{\mathcal{P}^{(2)}}\right) .
\end{aligned}
$$


The Hamiltonian constraint reduces to

$$
\frac{\left[\dot{q}_{0}^{2}-\mathcal{Q}_{0}^{2}\right]}{q_{0}^{2}}+\psi_{1} \frac{\left[\dot{q}_{(1)}^{2}-\mathcal{P}^{(1) 2}\right]}{q_{(1)}^{2}}+\psi_{2} \frac{\left[\dot{q}_{(2)}^{2}-\mathcal{P}^{(2) 2}\right]}{q_{(2)}^{2}}=c^{2},
$$

in terms of the independent fields, where $\psi_{1}, \psi_{2}$ are determined by the charges through (4.22). Substituting in the solution, we obtain

$$
\left(B_{0}\right)^{2}+\psi_{1}\left(B^{(1)}\right)^{2}+\psi_{2}\left(B^{(2)}\right)^{2}=c^{2} .
$$

\subsubsection{The quantum-deformed $S T U$-model}

We conclude this section with a specific example namely the quantum-deformed $S T U$ model with prepotential

$$
F=-\frac{Y^{1} Y^{2} Y^{3}+a\left(Y^{1}\right)^{3}}{Y^{0}}
$$

This is a particular model where the block $H^{\alpha^{\prime} \beta^{\prime}}$ does not sub-divide, so that we only have the two-block structure of generic very special prepotentials. While all formulas given in this section follow straightforwardly from our general results, we give various formulas explicitly for reference, since this model has many applications.

The Hesse potential for PI configurations is

$$
H(u, v)=-4\left[-v_{0}\left(u^{1} u^{2} u^{3}+a\left(u^{1}\right)^{3}\right)\right]^{\frac{1}{2}} \Leftrightarrow H\left(q_{a}\right)=-\frac{1}{4} q_{0}^{-\frac{1}{2}}\left[q_{5} q_{6} q_{7}+a q_{5}^{3}\right]^{-\frac{1}{2}} .
$$

This implies

$$
\tilde{H}\left(q_{a}\right) \sim \log q_{0}+\log \left(q_{5} q_{6} q_{7}+a q_{5}^{3}\right),
$$

so that from (4.3) we find $\tilde{H}^{a b}$ has the following block decomposition

$$
\tilde{H}^{a b}=\frac{\partial^{2}(-\tilde{H})}{\partial q_{a} \partial q_{b}}=\left(\begin{array}{cc|ccc|ccc}
\frac{1}{4} q_{0}^{-2} & 0 & 0 & 0 & 0 & 0 & 0 & 0 \\
\hline 0 & * & * & * & * & 0 & 0 & 0 \\
0 & * & * & * & * & 0 & 0 & 0 \\
0 & * & * & * & * & 0 & 0 & 0 \\
0 & * & * & * & * & 0 & 0 & 0 \\
\hline 0 & 0 & 0 & 0 & 0 & \tilde{H}^{55} & \tilde{H}^{56} & \tilde{H}^{57} \\
0 & 0 & 0 & 0 & 0 & \tilde{H}^{65} & \tilde{H}^{66} & \tilde{H}^{67} \\
0 & 0 & 0 & 0 & 0 & \tilde{H}^{75} & \tilde{H}^{76} & \tilde{H}^{77}
\end{array}\right) .
$$

We take the independent scalars to be $q_{0}$ and $q_{(1)}$, where

$$
q_{(1)}:=q_{5}=\xi_{6}^{-1} q_{6}=\xi_{7}^{-1} q_{7} .
$$

The solution has a full set of $n+1$ charges $\mathcal{Q}_{0}, \mathcal{P}^{A}$, and we choose to express the scalar fields in terms of the two charges $\mathcal{Q}_{0}$ and $\mathcal{P}^{(1)}:=\mathcal{P}^{1}$. The independent fields satisfy the equations of motion

$$
\ddot{q}_{0}-\frac{\left[\dot{q}_{0}^{2}-\mathcal{Q}_{0}^{2}\right]}{q_{0}}=0, \quad \ddot{q}_{(1)}-\frac{\left[\dot{q}_{(1)}^{2}-\mathcal{P}^{(1)^{2}}\right]}{q_{(1)}}=0,
$$


with explicit solution

$$
\begin{aligned}
q_{0} & = \pm \frac{-\mathcal{Q}_{0}}{B_{0}} \sinh \left(B_{0} \tau+B_{0} \frac{h_{0}}{\mathcal{Q}_{0}}\right) \\
q_{(1)} & = \pm \frac{\mathcal{P}^{(1)}}{B^{(1)}} \sinh \left(B^{(1)} \tau+B^{(1)} \frac{h^{(1)}}{\mathcal{P}^{(1)}}\right) .
\end{aligned}
$$

Substituting this solution into the Hamiltonian constraint

$$
\frac{1}{4} \frac{\left[\dot{q}_{0}^{2}-\mathcal{Q}_{0}^{2}\right]}{q_{0}^{2}}+\frac{3}{4} \frac{\left[\dot{q}_{(1)}^{2}-\mathcal{P}^{(1)^{2}}\right]}{q_{(1)}^{2}}=c^{2},
$$

gives

$$
\frac{1}{4}\left(B_{0}\right)^{2}+\frac{3}{4}\left(B^{(1)}\right)^{2}=c^{2}
$$

Observe that the coefficients on the left hand side sum to one. As we already remarked below equation (4.23), we will see in section 5.4 that this is a condition which is related to the regularity of the lifted four-dimensional solution.

We can use this example to demonstrate that setting scalar fields belonging to the same block proportional to one another is a consistent truncation: if we use (4.39) to reduce the Hesse potential to

$$
H\left(q_{0}, q_{(1)}\right)=-\frac{\beta}{2} q_{0}^{-\frac{1}{2}} q_{(1)}^{-\frac{3}{2}}, \quad \beta=\frac{1}{2}\left(\xi_{6} \xi_{7}+a\right)^{-1 / 2},
$$

then using (4.3) we find

$$
\tilde{H}^{00}=\frac{1}{4} q_{0}^{-2}, \quad \tilde{H}^{(1)(1)}=\frac{3}{4} q_{(1)}^{-2},
$$

as well as

$$
\tilde{H}_{00} \partial^{0} \tilde{H}^{00}=-2 q_{0}^{-1}, \quad \tilde{H}_{(1)(1)} \partial^{(1)} \tilde{H}^{(1)(1)}=-2 q_{0}^{-1},
$$

From these relations we obtain the equations of motion (4.40), which thus follow from a one-dimensional sigma model of the form (3.20) with Hesse potential (4.44) and the Hamiltonian constraint (4.43).

\section{$5 \quad$ Lifting to four dimensions}

Having obtained three-dimensional instanton solutions, we now need to lift them back to four dimensions and identify the subset which corresponds to black hole solutions with regular horizons. Let us therefore explain how one may read off the four-dimensional metric $g_{\hat{\mu} \hat{\nu}}^{(4)}$, gauge fields $F_{\hat{\mu} \hat{\nu}}^{I}$ and PSK scalar fields $z^{A}$ from the fields $g_{\mu \nu}^{(3)}, q_{a}, \hat{q}_{a}$, which we used to solve the three-dimensional equations of motion in section 4 . This essentially reverses the dimensional reduction procedure and transformation to dual coordinates given in sections 2.3 and A.2. We will restrict ourselves to spherically symmetric and purely imaginary field configurations of models with prepotentials of the form (3.2). 


\subsection{General formulas for lifted solutions}

We begin by determining the KK-scalar $e^{\phi}$ in terms of $q_{a}$. As seen in (2.12), this is proportional to the Hesse potential:

$$
e^{\phi}=-2 H\left(q^{a}\left(q_{b}\right)\right)
$$

where

$$
H\left(q^{a}\left(q_{b}\right)\right)=-\frac{1}{(2 \lambda+2)}\left[\left(\frac{-q_{0}}{\lambda}\right)^{\lambda} f\left(q_{n+2}, \ldots, q_{2 n+1}\right)\right]^{-\frac{1}{\lambda+1}} .
$$

One may then read off the four-dimensional metric $g^{(4)}$ from (2.8), using that for static solutions we can set $V_{\mu}=0$. Note that the three-dimensional part of the metric is fixed to be (3.16) by the Einstein equations after imposing spherical symmetry.

We now turn to the gauge fields. First we will need the four-dimensional complex gauge coupling matrix

$$
\mathcal{N}_{I J}=\bar{F}_{I J}+i \frac{N_{I K} Y^{K} N_{J L} Y^{L}}{Y^{M} N_{M N} Y^{N}}, \quad N_{I J}=2 \operatorname{Im} F_{I J} .
$$

From (3.10) we obtain

$$
\begin{aligned}
\left.F_{00}\right|_{\mathrm{PI}} & =i(-1)^{\lambda} \lambda(\lambda+1) \frac{f(u)}{\left(x^{0}\right)^{\lambda+2}},\left.\quad F_{0 A}\right|_{\mathrm{PI}}=(-1)^{\lambda+1} \lambda \frac{f_{A}(u)}{\left(x^{0}\right)^{\lambda+1}}, \\
\left.F_{A B}\right|_{\mathrm{PI}} & =i(-1)^{\lambda+1} \frac{f_{A B}(u)}{\left(x^{0}\right)^{\lambda}}
\end{aligned}
$$

which shows in particular that $F_{00}, F_{A B}$ are imaginary while $F_{0 A}$ are real on PI configurations. Next we obtain

$$
\left.N_{00}\right|_{\mathrm{PI}}=2(-1)^{\lambda} \lambda(\lambda+1) \frac{f(u)}{\left(x^{0}\right)^{\lambda+2}},\left.\quad N_{0 A}\right|_{\mathrm{PI}}=0,\left.\quad N_{A B}\right|_{\mathrm{PI}}=2(-1)^{\lambda+1} \frac{f_{A B}(u)}{\left(x^{0}\right)^{\lambda}} .
$$

Further useful formulae are

$$
\begin{aligned}
\left.\left(N_{0 I} Y^{I}\right)\right|_{\mathrm{PI}} & =2(-1)^{\lambda} \lambda(\lambda+1) \frac{f(u)}{\left(x^{0}\right)^{\lambda+1}},\left.\quad\left(N_{A I} Y^{I}\right)\right|_{\mathrm{PI}}=2 i(-1)^{\lambda+1}(\lambda+1) \frac{f_{A}(u)}{\left(x^{0}\right)^{\lambda}}, \\
\left.\left(Y^{I} N_{I J} Y^{J}\right)\right|_{\mathrm{PI}} & =4(-1)^{\lambda}(\lambda+1)^{2} \frac{f(u)}{\left(x^{0}\right)^{\lambda}} .
\end{aligned}
$$

Using these it is straightforward to verify

$$
\begin{gathered}
\left.\mathcal{N}_{00}\right|_{\mathrm{PI}}=i \lambda(-1)^{\lambda+1} \frac{f(u)}{\left(x^{0}\right)^{\lambda+2}},\left.\quad \mathcal{N}_{0 A}\right|_{\mathrm{PI}}=0, \\
\left.\mathcal{N}_{A B}\right|_{\mathrm{PI}}=i(-1)^{\lambda} \frac{1}{\left(x^{0}\right)^{\lambda}}\left(f_{A B}(u)-\frac{f_{A}(u) f_{B}(u)}{f(u)}\right),
\end{gathered}
$$

which shows in particular that $\mathcal{N}_{I J}$ is purely imaginary on PI configurations. Note that this does not follow automatically from the reality properties that we have imposed. The conditions by themselves allow real elements $\left.\mathcal{N}_{0 A}\right|_{\text {PI }}$, and it requires an explicit calculation 
to see that these matrix elements are in fact zero. The actual computation of the fourdimensional gauge fields is more easily performed using the real version

$$
\left(\hat{H}_{a b}\right)=\left(\begin{array}{cc}
\mathcal{I}+\mathcal{R} \mathcal{I}^{-1} \mathcal{R} & -\mathcal{R} \mathcal{I}^{-1} \\
-\mathcal{I}^{-1} \mathcal{R} & \mathcal{I}^{-1}
\end{array}\right), \quad \mathcal{N}_{I J}=\mathcal{R}_{I J}+i \mathcal{I}_{I J}
$$

of the gauge coupling matrix. As shown above we have $\mathcal{R}_{I J}=0$ on PI configurations. The electric components of the four-dimensional gauge fields are determined by $\dot{\hat{q}}_{a}=K_{a}$ to be

$$
\left(\begin{array}{c}
F_{t \tau}^{I} \\
G_{I \mid t \tau}
\end{array}\right)=\left(\begin{array}{c}
-\dot{\zeta}^{I} \\
-\dot{\tilde{\zeta}}_{I}
\end{array}\right)=-2\left(\dot{\hat{q}}^{a}\right)=-2\left(\tilde{H}^{a b} K_{b}\right) .
$$

Using the block structure of $\tilde{H}^{a b}$ as well as that

$$
K_{a}=\left(K_{0}, 0, \ldots, 0, K_{n+2}, \ldots, K_{2 n+1}\right)=\left(-\mathcal{Q}_{0}, 0, \ldots, 0, \mathcal{P}^{A}\right)
$$

we obtain:

$$
\begin{aligned}
2 F_{t \tau}^{0} & =-2 \tilde{H}^{00} K_{0}, \quad F_{t \tau}^{A}=0, \quad G_{0 \mid t \tau}=0, \\
G_{A \mid t \tau} & =-2 \tilde{H}^{A+(n+1), B+(n+1)} K_{B+(n+1)}, \quad A, B=1, \ldots, n .
\end{aligned}
$$

For PI field configurations, where $\mathcal{N}_{I J}$ is purely imaginary, the field strength and dual field strength are related by

$$
F_{\hat{\mu} \hat{\nu}}^{I}=-\frac{i}{2} \sqrt{\left|\operatorname{det} g^{(4)}\right|} \epsilon_{\hat{\mu} \hat{\nu} \hat{\rho} \hat{\sigma}} g^{\hat{\rho} \hat{\alpha}} g^{\hat{\sigma} \hat{\beta}} \mathcal{N}^{I J} G_{J \mid \hat{\alpha} \hat{\beta}} .
$$

Using this relation we can relate the electric components $G_{I \mid t \tau}$ of the dual gauge fields to the magnetic components $F_{\theta \phi}^{I}$ of the gauge fields. This requires computing the inverse gauge coupling matrix $\mathcal{N}^{I J}$. We use (5.3) with $\mathcal{R}_{I J}=0$ together with the relation [22]

$$
\hat{H}_{a b}=H \tilde{H}_{a b}+\frac{2}{H} \Omega_{a c} q^{c} \Omega_{b d} q^{d} .
$$

Evaluating this for the block where $a, b,=n+1, \ldots, 2 n+1$, we obtain:

$$
\begin{aligned}
\left.\mathcal{N}^{00}\right|_{\mathrm{PI}} & =i\left(H \tilde{H}_{n+1, n+1}+\frac{2}{H} x^{0} x^{0}\right),\left.\quad \mathcal{N}^{0 A}\right|_{\mathrm{PI}}=0, \\
\left.\mathcal{N}^{A B}\right|_{\mathrm{PI}} & =i H \tilde{H}_{A+(n+1), B+(n+1)} .
\end{aligned}
$$

Using this as well as the explicit form of the four-dimensional space-time metric $g^{(4)}$ given in (2.8) with $V_{\mu}=0$ and $g^{(3)}$ given by (3.16) we obtain:

$$
F_{\theta \phi}^{0}=0, \quad F_{\theta \phi}^{A}=-\frac{1}{2} K_{A+(n+1)} \sin \theta=-\frac{1}{2} \mathcal{P}^{A} \sin \theta=-\frac{1}{2} \dot{\hat{q}}_{A+(n+1)} \sin \theta .
$$

The results for the field strength can thus be summarised as

$$
F^{0}=-2 \tilde{H}^{00} \dot{\hat{q}}_{0} d t \wedge d \tau, \quad F^{A}=-\frac{1}{2} \dot{\hat{q}}_{A+(n+1)} \sin \theta d \theta \wedge d \phi,
$$


with

$$
\dot{\hat{q}}_{0}=K_{0}=-\mathcal{Q}_{0}, \quad \dot{\hat{q}}_{A+(n+1)}=K_{A+(n+1)}=\mathcal{P}_{A} .
$$

The complex scalar fields $X^{I}=e^{-\phi / 2} Y^{I}$ are given by

$$
X^{0}=(-2 H)^{-1 / 2} x^{0}, \quad X^{A}=i(-2 H)^{-1 / 2} u^{A} .
$$

Since we know from section 3 that $^{13}$

$$
x_{0}=\phi_{x}\left(\frac{\lambda f(u)}{v_{0}}\right)^{\frac{1}{\lambda+1}}=\phi_{x}(-1)^{\lambda} H\left(\frac{\lambda f\left(q_{\alpha^{\prime}}\right)}{-q_{0}}\right)^{\frac{1}{\lambda+1}}
$$

we can express $X^{0}$ in terms of $q_{a}$ by

$$
X^{0}=\tilde{\phi}_{x} \sqrt{-\frac{H}{2}}\left(\frac{\lambda f\left(q_{\alpha^{\prime}}\right)}{-q_{0}}\right)^{\frac{1}{\lambda+1}}
$$

where

$$
\tilde{\phi}_{x}:=(-1)^{\lambda+1} \phi_{x}=\left\{\begin{array}{ll}
\operatorname{sgn}\left(x^{0}\right) & \text { for } \lambda+1 \text { even } \\
1 & \text { for } \lambda+1 \text { odd }
\end{array}, \quad \tilde{\phi}_{x}^{-1}=\tilde{\phi}_{x} .\right.
$$

Since

$$
X^{A}=-i \sqrt{-\frac{H}{2}} q_{A+(n+1)}
$$

the four-dimensional scalars are

$$
z^{A}=-i \tilde{\phi}_{x} q_{A+(n+1)}\left(\frac{-q_{0}}{\lambda f\left(q_{\alpha^{\prime}}\right)}\right)^{\frac{1}{\lambda+1}} .
$$

This is purely imaginary, as required by the PI conditions, provided that the conditions explained between (3.11) and (3.13) are satisfied. To see this explicitly, remember how these conditions look in terms of $q_{a}$ :

- If $\lambda+1$ is odd, then there is no sign ambiguity in $x^{0}$ but reality of the Kaluza-Klein scalar implies $f(u)>0 \Leftrightarrow f\left(q_{\alpha^{\prime}}\right)>0$. In this case $\phi_{x}=-1, \tilde{\phi}_{x}=1$.

- If $\lambda+1$ is even, then we need to impose $v_{0} f(u)>0 \Leftrightarrow-q_{0} f\left(q_{\alpha^{\prime}}\right)>0$. We can have two different signs: if $v_{0}>0 \Leftrightarrow q_{0}>0$, then $x^{0}<0$ so that $\tilde{\phi}_{x}=-1$, whereas if $v_{0}<0 \Leftrightarrow q_{0}<0$, then $x^{0}>0$ so that $\tilde{\phi}_{x}=1$.

In either case the root is manifestly real, and thus $z^{A}$ is manifestly purely imaginary. Also note that $z^{A}$ are homogeneous of degree zero in $q_{a}$.

\footnotetext{
${ }^{13}$ Some care is required with regard to signs in the following. Remember that $H<0$, so that $\left(H^{\lambda+1}\right)^{1 /(\lambda+1)}=(-1)^{\lambda} H$.
} 


\subsection{Black hole regularity conditions}

Not all four-dimensional solutions (5.1)-(5.5) obtained by lifting three-dimensional instanton solutions describe black hole spacetimes. We regard four-dimensional solutions to be genuine black holes if the following three regularity conditions are met:

(i) there exists an outer horizon, $E$, of finite area,

(ii) the physical (PSK) scalar fields, $z^{A}$, take finite values on $E$,

(iii) the metric is asymptotically Minkowski.

The third condition is checked by evaluating $e^{\phi}$ at radial infinity $\left(\tau \rightarrow 0^{+}\right)$, and the second by evaluating $z^{A}$ at the horizon $(\tau \rightarrow+\infty)$. For the first condition one must use the formula for the area

$$
A=\int_{E} \operatorname{vol}_{E}=\lim _{\tau \rightarrow+\infty} \int_{E_{\tau}} \sqrt{\operatorname{det} g^{(2)}} d \Omega_{(2)},
$$

where $g^{(2)}$ is the pullback of the four-dimensional metric $g^{(4)}$ to the two-dimensional surface $E_{\tau}$ given by $t, \tau=$ const. $E_{\tau}$ is independent of $t$, and the event horizon $E$ is obtained by $\tau \rightarrow+\infty$.

\subsection{Diagonal models}

We will now turn to explicit examples, starting with diagonal models, i.e. models with prepotential of the form (4.1). Three-dimensional instanton solutions were found in the previous section, described by (4.13) and (4.15), which we shall now lift to four dimensions. We may use (5.1) and (5.2) to write the KK-scalar for this solution as

$$
e^{\phi}=\frac{1}{(\lambda+1)}\left[\left(\frac{-q_{0}}{\lambda}\right)^{\lambda}\left(q_{n+2} \ldots q_{2 n+1}\right)^{\frac{\lambda+2}{n}}\right]^{-\frac{1}{\lambda+1}} .
$$

Using (2.8) and (3.16), we can insert the above warp factor to ascertain the following four-dimensional metric

$$
\begin{aligned}
d s_{4}^{2}= & -\frac{1}{(\lambda+1)}\left[\left(\frac{-q_{0}}{\lambda}\right)^{\lambda}\left(q_{n+2} \ldots q_{2 n+1}\right)^{\frac{\lambda+2}{n}}\right]^{-\frac{1}{\lambda+1}} d t^{2}+ \\
& (\lambda+1)\left[\left(\frac{-q_{0}}{\lambda}\right)^{\lambda}\left(q_{n+2} \ldots q_{2 n+1}\right)^{\frac{\lambda+2}{n}}\right]^{\frac{1}{\lambda+1}}\left(\frac{c^{4}}{\sinh ^{4}(c \tau)} d \tau^{2}+\frac{c^{2}}{\sinh ^{2}(c \tau)} d \Omega_{(2)}^{2}\right) .
\end{aligned}
$$

The gauge fields are given by

$$
F^{0}=\frac{\lambda}{(\lambda+1)} \frac{\mathcal{Q}_{0}}{q_{0}^{2}} d t \wedge d \tau, \quad F^{A}=-\frac{1}{2} \mathcal{P}^{A} \sin \theta d \theta \wedge d \phi,
$$

and scalar fields by

$$
z^{A}=-i \tilde{\phi}_{x} q_{n+1+A}\left[\left(\frac{-q_{0}}{\lambda}\right) \frac{1}{\left(q_{n+2} \ldots q_{2 n+1}\right)^{\frac{\lambda+2}{n}}}\right]^{\frac{1}{\lambda+1}} .
$$


This is the most general stationary field configuration that is spherically symmetric and purely imaginary. Along with the charges $\mathcal{Q}_{0}, \mathcal{P}^{1}, \ldots, \mathcal{P}^{n}$ there are $2 n+2$ further free parameters in this solution: $B_{0}, B^{1}, \ldots, B^{n}$ and $h_{0}, h^{1}, \ldots, h^{n}$, where we interpret $c$ as a dependent parameter, which is determined by (4.15).

We would like to determine for which choices of parameters this solution corresponds to a genuine black hole. The area of the horizon is given by

$$
A=4 \pi \lim _{\tau \rightarrow+\infty}(\lambda+1)\left[\left(\frac{-q_{0}}{\lambda}\right)^{\lambda}\left(q_{n+2} \ldots q_{2 n+1}\right)^{\frac{\lambda+2}{n}}\right]^{\frac{1}{\lambda+1}} \frac{c^{2}}{\sinh ^{2}(c \tau)} .
$$

Expanding the solution of the scalar fields (4.13) in terms of exponentials tells us that the highest order term in the numerator is

$$
\exp \left[\left(\frac{\lambda}{\lambda+1} B_{0}+\frac{\lambda+2}{(\lambda+1) n} B^{1}+\cdots+\frac{\lambda+2}{(\lambda+1) n} B^{n}\right) \tau\right] .
$$

Meanwhile, the highest order term in the denominator is given by $e^{2 c \tau}$. In order to obtain a finite area these terms must exactly match one-another, which places the following constraint on the integration constants

$$
\frac{\lambda}{\lambda+1} B_{0}+\frac{\lambda+2}{(\lambda+1) n} B^{1}+\cdots+\frac{\lambda+2}{(\lambda+1) n} B^{n}=2 c .
$$

We also impose that the physical scalar fields $z^{A}$ take finite values on the horizon. In the limit $\tau \rightarrow+\infty$ the $q_{a}$ scalars behave as $q_{0} \sim e^{B_{0} \tau}, q_{n+2} \sim e^{B^{1} \tau}, \ldots, q_{2 n+1} \sim e^{B^{n} \tau}$, and so the only way to guarantee that the $z^{A}$ remain finite on the horizon is to set

$$
B_{0}=B^{1}=\cdots=B^{n} .
$$

If we combine this with the finite horizon constraint (5.12), we see that $B=c$ i.e. the integration constants satisfy

$$
B_{0}=B^{1}=\cdots=B^{n}=c .
$$

At this point, we can rewrite the solution in (4.13) for the scalar fields as

$$
q_{0}=-\frac{\mathcal{Q}_{0}}{c} \sinh \left(c \tau+c \frac{h_{0}}{\mathcal{Q}_{0}}\right), \quad q_{\alpha^{\prime}}=\frac{\mathcal{P}^{\alpha^{\prime}}}{c} \sinh \left(c \tau+c \frac{h^{\alpha^{\prime}}}{\mathcal{P}^{\alpha^{\prime}}}\right),
$$

$\alpha^{\prime}=n+2, \ldots, 2 n+1$. We also impose that the solution is asymptotically flat. From (2.8) we see that in order to obtain Minkowski space at radial infinity we need to ensure that $e^{\phi} \rightarrow 1$. By (5.7) this places one more constraint on the integration constants

$$
\left[\frac{\mathcal{Q}_{0}}{\lambda c} \sinh \left(\frac{c h_{0}}{\mathcal{Q}_{0}}\right)\right]^{\lambda}\left[\frac{\mathcal{P}^{1}}{c} \sinh \left(\frac{c h^{1}}{\mathcal{P}^{1}}\right) \ldots \frac{\mathcal{P}^{n}}{c} \sinh \left(\frac{c h^{n}}{\mathcal{P}^{n}}\right)\right]^{\frac{\lambda+2}{n}}=(\lambda+1)^{-(\lambda+1)} .
$$

It's worth noting that the constrained scalars above automatically satisfy the Hamiltonian constraint (4.14). The solution described by (5.15) satisfies conditions (i), (ii) and 
(iii) and therefore describes a black hole. Other than the charges, the solution is described by the $n+2$ parameters $h_{0}, h^{1}, \ldots, h^{n}$ and $c$. These are subject to one algebraic constraint (5.16). This leaves a total of $n+1$ independent parameters in the solution for $q_{a}$, $a=0, n+2, \ldots, 2 n+1$, which is consistent with and suggestive of the existence of a first order rewriting of the equations of motion. We will come back to this in section 6 .

For the interpretation as a black hole, it is convenient to replace the 'affine' radial coordinate $\tau$, by the radial coordinate $\rho$ defined in (3.17). This rewriting will make explicit that the four-dimensional metric (5.8) is a deformation of the Reissner-Nordström metric, and will allow us to express the solution in terms of harmonic functions.

To demonstrate the rewriting of the scalar fields, consider $q_{0}$ :

$$
\begin{aligned}
q_{0} & =-\frac{\mathcal{Q}_{0}}{c} \sinh \left(c \tau+c \frac{h_{0}}{\mathcal{Q}_{0}}\right) \\
& =-W^{-\frac{1}{2}}\left[\frac{\mathcal{Q}_{0}}{c} \sinh \left(c \frac{h_{0}}{\mathcal{Q}_{0}}\right)+\mathcal{Q}_{0} e^{-c \frac{h_{0}}{\mathcal{Q}_{0}}} \frac{1}{\rho}\right] \\
& =\left(\frac{\lambda^{\lambda}}{(\lambda+1)^{\lambda+1}}\right)^{\frac{1}{2 \lambda+2}} \frac{\mathcal{H}_{0}}{W^{\frac{1}{2}}},
\end{aligned}
$$

where

$$
\mathcal{H}_{0}=-\left(\frac{(\lambda+1)^{\lambda+1}}{\lambda^{\lambda}}\right)^{\frac{1}{2 \lambda+2}}\left[\frac{\mathcal{Q}_{0}}{c} \sinh \left(c \frac{h_{0}}{\mathcal{Q}_{0}}\right)+\mathcal{Q}_{0} e^{-c \frac{h_{0}}{\mathcal{Q}_{0}}} \frac{1}{\rho}\right]
$$

is a harmonic function. The prefactors have been chosen such that this and the following expressions are as simple as possible, while allowing $\lambda$ to be general. Similarly we can express the other scalars as ratios of harmonic functions:

$$
q_{n+1+A}=\left(\frac{\lambda^{\lambda}}{(\lambda+1)^{\lambda+1}}\right)^{\frac{1}{2 \lambda+2}} \frac{\mathcal{H}^{A}}{W^{\frac{1}{2}}}
$$

where

$$
\mathcal{H}^{A}=\left(\frac{(\lambda+1)^{\lambda+1}}{\lambda^{\lambda}}\right)^{\frac{1}{2 \lambda+2}}\left[\frac{\mathcal{P}^{A}}{c} \sinh \left(c \frac{h^{A}}{\mathcal{P}^{A}}\right)+\mathcal{P}^{A} e^{-c \frac{h^{A}}{\mathcal{P}^{A}}} \frac{1}{\rho}\right] .
$$

The four-dimensional scalar fields can now be expressed in terms of the harmonic functions as

$$
z^{A}=-i \tilde{\phi}_{x} \lambda^{-\frac{1}{\lambda+1}} \mathcal{H}_{A}\left(\frac{-\mathcal{H}_{0}}{\left(\mathcal{H}^{1} \cdots \mathcal{H}^{n}\right)^{\frac{\lambda+2}{n}}}\right)^{\frac{1}{\lambda+1}}
$$

Substituting our results into (5.7), we find the Kaluza-Klein scalar can be expressed as

$$
e^{\phi}=\frac{W}{\left[\left(-\mathcal{H}_{0}\right)^{\lambda}\left(\mathcal{H}^{1} \ldots \mathcal{H}^{n}\right)^{\frac{\lambda+2}{n}}\right]^{\frac{1}{\lambda+1}}},
$$


and the four-dimensional metric becomes

$$
\begin{aligned}
d s_{4}^{2}= & -\frac{W}{\left[\left(-\mathcal{H}_{0}\right)^{\lambda}\left(\mathcal{H}^{1} \ldots \mathcal{H}^{n}\right)^{\frac{\lambda+2}{n}}\right]^{\frac{1}{\lambda+1}}} d t^{2} \\
& +\left[\left(-\mathcal{H}_{0}\right)^{\lambda}\left(\mathcal{H}^{1} \ldots \mathcal{H}^{n}\right)^{\frac{\lambda+2}{n}}\right]^{\frac{1}{\lambda+1}}\left(\frac{d \rho^{2}}{W}+\rho^{2} d \Omega_{(2)}^{2}\right) .
\end{aligned}
$$

Writing the metric this way draws parallels with the metric for extremal black hole solutions which can always be written in terms of harmonic functions, and which is recovered for $c \rightarrow$ 0 . Moreover, the solution for the scalar fields takes, when expressed in terms of harmonic functions, exactly the same form as for extreme solutions. However the coefficients of the harmonic functions change, and depend on the non-extremality parameter $c$, as made explicit in (5.19)

The non-extremal Reissner-Nordström metric is recovered by setting the harmonic functions $\mathcal{H}_{0}, \mathcal{H}^{A}$ proportional to one-another, in which case the solution carries $n+1$ independent charges $\mathcal{Q}_{0}, \mathcal{P}^{A}$, while the scalars $z^{A}$ are constant. This solution corresponds to the universal solution described in section 4.2 which exists irrespective of a block decomposition.

\subsubsection{STU-like models}

A one-parameter subclass of diagonal models is given by setting $\lambda=1$ in (4.1), resulting in prepotentials of the form

$$
F=\frac{\left(Y^{1} \ldots Y^{n}\right)^{\frac{3}{n}}}{Y^{0}} .
$$

Since the well-known $S T U$ model corresponds to the particular choice $n=3$, we will refer to this class of models as $S T U$-like. For PI field configurations it follows from (5.2) that the Hesse potential takes the form

$$
H\left(q_{a}\right)=-\frac{1}{4}\left[-q_{0}\left(q_{1} \ldots q_{n}\right)^{\frac{3}{n}}\right]^{-\frac{1}{2}} .
$$

Explicit expressions for the solution can be obtained by substituting $\lambda=1$ into (4.13) and (3.24), with the integration constants subject to the constraint (4.15). The fourdimensional metric is given by

$$
\begin{aligned}
d s_{4}^{2}= & -\frac{1}{2} \frac{1}{\sqrt{-q_{0}\left(q_{n+1} \ldots q_{2 n+1}\right)^{\frac{3}{n}}}} d t^{2} \\
& +2 \sqrt{-q_{0}\left(q_{n+1} \ldots q_{2 n+1}\right)^{\frac{3}{n}}}\left(\frac{c^{4}}{\sinh ^{4} c \tau} d \tau^{2}+\frac{c^{2}}{\sinh ^{2} c \tau} d \Omega_{(2)}^{2}\right) .
\end{aligned}
$$

The gauge fields are given by

$$
F^{0}=\frac{1}{2} \frac{\mathcal{Q}_{0}}{q_{0}^{2}} d t \wedge d \tau, \quad F^{A}=-\frac{1}{2} \mathcal{P}^{A} \sin \theta d \theta \wedge d \phi
$$


and the scalar fields by

$$
z^{A}=-i \tilde{\phi}_{x} q_{n+1+A} \sqrt{\frac{-q_{0}}{\left(q_{n+2} \ldots q_{2 n+1}\right)^{\frac{3}{n}}}} .
$$

For this solution to describe a black hole the scalar fields take the restricted form (5.15), and so the number of integration constants reduces from $2 n+2$ down to $n+1$. In this case one may re-express the four-dimensional metric in terms of harmonic functions and the isotropic radial coordinate, $\rho$, as

$$
d s_{4}^{2}=-\frac{W}{\sqrt{-\mathcal{H}_{0}\left(\mathcal{H}^{1} \ldots \mathcal{H}^{n}\right)^{\frac{3}{n}}}} d t^{2}+\sqrt{-\mathcal{H}_{0}\left(\mathcal{H}^{1} \ldots \mathcal{H}^{n}\right)^{\frac{3}{n}}}\left(\frac{d \rho^{2}}{W}+\rho^{2} d \Omega_{(2)}^{2}\right),
$$

where $W, \mathcal{H}_{0}$ and $\mathcal{H}^{A}$ are harmonic functions with respect to the flat metric on $\mathbb{R}^{3}$. We recall that $W=1-\frac{2 c}{\rho}=e^{-2 c \tau}$ whilst $\mathcal{H}_{0}, \mathcal{H}^{A}$ are obtained by substituting $\lambda=1$ into the expressions in section 5.3. The four-dimensional scalar fields are given by

$$
z^{A}=-i \tilde{\phi}_{x} \mathcal{H}_{A} \sqrt{\frac{-\mathcal{H}_{0}}{\left(\mathcal{H}^{1} \cdots \mathcal{H}^{n}\right)^{\frac{3}{n}}}} .
$$

For $\lambda+1$ even there are two possible choices for $\tilde{\phi}_{x}$ in (5.23). Note, however, that this does not necessarily imply that we have two physically inequivalent solutions. The reason is that the sign of $\tilde{\phi}_{x}$ can be correlated with that of $q_{(n+1)+A}$. To decide the sign (and in fact the allowed range) of $z^{A}$ is a model dependent problem. Let us illustrate this with the explicit example of the $S T U$ model, where $n=3$. This solution has previous appeared in [56]. In this case we know that the manifold parametrised by the physical scalars $z^{A}$ is isometric to three copies of the Poincaré half plane. Since $u^{A}=0, A=1,2,3$ corresponds to the boundary, we can take each $u^{A}$ to be either positive or negative. It is convenient to choose the same sign for all $u^{A}$. It is straightforward to verify that if we either take all $u^{A}$ to be positive, or all $u^{A}$ to be negative, the only solutions consistent with all conditions are such that $\operatorname{Im} z^{A}<0$, that is all scalars $z^{A}$ take values in lower half plane. The standard supergravity fields with positive real part are then $S=i z^{1}, T=i z^{2}, U=i z^{3}$. The more conventional description of the $S T U$ model is obtained by including a minus sign in the definition of the prepotential. For $F=-\left(Y^{1} Y^{2} Y^{3}\right) / Y^{0}$ one finds by a similar analysis that taking $u^{A}>0$ (or $u^{A}<0$ ) for all $A=1,2,3$ leads to $\operatorname{Im} z^{A}>0$, and in this case the standard supergravity fields are $S=-i z^{1}, T=-i z^{2}, U=-i z^{3}$.

In general the choice of the prepotential determines a range of the scalar fields where the scalar metric is positive definite. The parameters of a solution should then be restricted such that scalar fields take only values within this range. This analysis is model dependent, and we will not further investigate it in this paper. The above example illustrates that it is relevant that for $\lambda$ odd one has two possible choices for the solution.

\subsubsection{The $F=i \frac{Y^{1} Y^{2} Y^{3} Y^{4}}{\left(Y^{0}\right)^{2}}$ model}

Let us next give one explicit example of a diagonal model with $\lambda>1$. For concreteness we choose the case $\lambda=2, n=4$, which is the minimal deviation from the $S T U$ model that is 
not very special. This model has prepotential

$$
F=i \frac{Y^{1} Y^{2} Y^{3} Y^{4}}{\left(Y^{0}\right)^{2}}
$$

and, using (3.14), we can show that for PI configurations, the Hesse potential assumes the form

$$
H\left(q_{a}\right)=-\frac{1}{6}\left[\frac{1}{4} q_{0}^{2} q_{6} q_{7} q_{8} q_{9}\right]^{-\frac{1}{3}} .
$$

Explicit expressions for the solution can be found by substituting $\lambda=2, n=4$ into (4.13) and (3.24), with the integration constants constrained by (4.15). Dimensionally lifting this solution produces the following four-dimensional metric

$$
\begin{aligned}
d s_{4}^{2}= & \frac{1}{3} \frac{1}{\sqrt[3]{\frac{1}{4} q_{0}^{2} q_{6} q_{7} q_{8} q_{9}}} d t^{2} \\
& +3 \sqrt[3]{\frac{1}{4} q_{0}^{2} q_{6} q_{7} q_{8} q_{9}}\left(\frac{c^{4}}{\sinh ^{4}(c \tau)} d \tau^{2}+\frac{c^{2}}{\sinh ^{2}(c \tau)} d \Omega_{(2)}^{2}\right) .
\end{aligned}
$$

The gauge fields are given by

$$
F^{0}=\frac{2}{3} \frac{\mathcal{Q}_{0}}{q_{0}^{2}} d t \wedge d \tau, \quad F^{A}=-\frac{1}{2} \mathcal{P}^{A} \sin \theta d \theta \wedge d \phi
$$

and the scalar fields by

$$
z^{A}=-i \tilde{\phi}_{x} q_{5+A} \sqrt[3]{\frac{-q_{0}}{2\left(q_{6} q_{7} q_{8} q_{9}\right)}}
$$

As before, for this solution to describe a black hole, the scalar fields must take the restricted form (5.15), which again reduces the number of integration constants from $2 n+2$ to $n+1$. We can then rewrite the four-dimensional metric in terms of harmonic functions and the isotropic radial coordinate, $\rho$, as

$$
\begin{aligned}
d s_{4}^{2}= & -\frac{W}{\sqrt[3]{\mathcal{H}_{0}^{2} \mathcal{H}^{1} \mathcal{H}^{2} \mathcal{H}^{3} \mathcal{H}^{4}}} d t^{2} \\
& +\sqrt[3]{\mathcal{H}_{0}^{2} \mathcal{H}^{1} \mathcal{H}^{2} \mathcal{H}^{3} \mathcal{H}^{4}}\left(\frac{d \rho^{2}}{W}+\rho^{2} d \Omega_{(2)}^{2}\right)
\end{aligned}
$$

where $W$ is a harmonic function given by $W=1-\frac{2 c}{\rho}=e^{-2 c \tau}$ and $\mathcal{H}_{0}, \mathcal{H}^{A}$ are harmonic functions obtained by substituting $\lambda=2, n=4$ into the expressions in section 5.3. The four-dimensional scalar fields are

$$
z^{A}=-i \tilde{\phi}_{x} \mathcal{H}_{A} \sqrt[3]{\frac{-\mathcal{H}_{0}}{2\left(\mathcal{H}^{1} \cdots \mathcal{H}^{4}\right)}}
$$




\subsection{Block diagonal models}

We shall now give a description of how to lift the three-dimensional instanton solutions of block diagonal models to four dimensions. For concreteness we consider the case where the bottom right block decomposes into two sub-blocks; one of size $k \times k$ and one of size $l \times l$ where $k \geq 1$ and $l=n-k$. Instanton solutions to such models were discussed in section 4.3 and are described by (4.30)-(4.34). Again, it is possible to use (5.1) and (5.2) to write the KK-scalar as

$$
e^{\phi}=\frac{1}{2 \sqrt{-q_{0} f_{1}\left(q_{(1)}\right) f_{2}\left(q_{(2)}\right)}},
$$

where we have decomposed the function $f$ appearing in (5.2) as discussed in (4.24) and set $\lambda=1$ as the models considered in section 4.3 are all obtainable from five dimensions. We can then use (2.8) and (3.16) to insert this warp factor into the four-dimensional metric as follows

$$
\begin{aligned}
d s_{4}^{2}= & -\frac{1}{2 \sqrt{-q_{0} f_{1}\left(q_{(1)}\right) f_{2}\left(q_{(2)}\right)}} d t^{2} \\
& +2 \sqrt{-q_{0} f_{1}\left(q_{(1)}\right) f_{2}\left(q_{(2)}\right)}\left(\frac{c^{4}}{\sinh ^{4}(c \tau)} d \tau^{2}+\frac{c^{2}}{\sinh (c \tau)} d \Omega_{(2)}^{2}\right) .
\end{aligned}
$$

From (5.4), the gauge fields are given by

$$
F^{0}=\frac{1}{2} \frac{\mathcal{Q}_{0}}{q_{0}^{2}} d t \wedge d \tau, \quad F^{A}=-\frac{1}{2} \mathcal{P}^{A} \sin \theta d \theta \wedge d \phi,
$$

and we make the observation that these are exactly the same as for the $S T U$-like models considered in section 5.3.1 (or indeed any diagonal model with $\lambda=1$ ). The only difference is that now the ratios between scalar fields belonging to the same block are determined by the ratios of the corresponding charges.

From (5.5), the scalar fields assume the form

$$
z^{A}=-i \tilde{\phi}_{x} q_{A+(n+1)} \sqrt{-\frac{q_{0}}{f_{1}\left(q_{(1)}\right) f_{2}\left(q_{(2)}\right)}}
$$

where $q_{A+(n+1)}$ is proportional to $q_{(1)}$ for $A=1, \ldots, k$ and proportional to $q_{(2)}$ for $A=$ $k+1, \ldots, k+l=n$. When viewing the non-extremality parameter $c$ as being determined by (4.34), we have, apart from the charges, 6 free parameters in the solution: $B_{0}, B^{(1)}, B^{(2)}$ and $h_{0}, h^{(1)}, h^{(2)}$.

Even without specifying the functions $f_{1}$ and $f_{2}$ we can see that these 6 parameters reduce to 3 when imposing the conditions that guarantee a regular black hole solution. The area of the horizon is

$$
A=8 \pi \lim _{\tau \rightarrow \infty} \sqrt{-q_{0} f_{1}\left(q_{(1)}\right) f_{2}\left(q_{(2)}\right)} \frac{c^{2}}{\sinh ^{2}(c \tau)} .
$$

From (4.19), we know that the product $f_{1}\left(q_{(1)}\right) f_{2}\left(q_{(2)}\right)$ is homogeneous degree three. Regardless of the individual degrees of homogeneity of $f_{1}$ and $f_{2}$, the requirement that the 
above area be finite together with the requirement that the $z^{A}$ take finite values on the horizon imply

$$
B_{0}=B^{(1)}=B^{(2)}=c .
$$

Moreover, substituting the solution back into the Hamiltonian constraint (4.33), we find that this is satisfied provided that $1+\psi_{1}+\psi_{2}=1 \Rightarrow \psi_{1}+\psi_{2}=0$, using that one of the three blocks in (4.18) only contains one scalar field $q_{0}$. For a general decomposition with $M$ blocks one finds that regularity requires $B^{(m)}=c$ for $m=1, \ldots, M$ so that the condition becomes (4.23).

Additionally, the requirement that $e^{\phi} \rightarrow 1$ as $\tau \rightarrow 0^{+}$, places one algebraic constraint on the parameters $h_{0}, h^{(1)}, h^{(2)}$. Altogether, these constraints reduce the 6 free parameters of the instanton solution to 3 free parameters.

If we know the functions $f_{1}$ and $f_{2}$ explicitly, then it is possible to rewrite the metric using the isotropic radial coordinate $\rho$, and with the warp factors being expressed as ratios of harmonic functions.

\subsubsection{The quantum deformed $S T U$ model}

As an explicit example of a block-diagonal model we consider the quantum deformed STU model with prepotential (4.35). In this case $\tilde{H}^{\alpha^{\prime} \beta^{\prime}}$ does not decompose into smaller blocks, so that this represents the generic situation for models obtainable from five dimensions. But we can adapt the formulae given above by choosing $f_{1}$ to have degree three and $f_{2}=1$.

The instanton solution is described by two independent scalars as seen in (4.41) and (4.43). We saw how to write the Hesse potential for such a solution in (4.44), from which we can use (5.1) to find the KK-scalar is

$$
e^{\phi}=\beta q_{0}^{-\frac{1}{2}} q_{(1)}^{-\frac{3}{2}}
$$

where $\beta$ was computed in (4.44). We can then substitute this into (2.8) to dimensionally lift the instanton solution to the following four dimensional metric

$$
d s_{4}^{2}=-\beta q_{0}^{-\frac{1}{2}} q_{(1)}^{-\frac{3}{2}} d t^{2}+\frac{1}{\beta q_{0}^{-\frac{1}{2}} q_{(1)}^{-\frac{3}{2}}}\left(\frac{c^{4}}{\sinh ^{4}(c \tau)} d \tau^{2}+\frac{c^{2}}{\sinh ^{2}(c \tau)} d \Omega_{(2)}^{2}\right) .
$$

The gauge fields take the same form as in (5.32) i.e.

$$
F^{0}=\frac{1}{2} \frac{\mathcal{Q}_{0}}{q_{0}^{2}} d t \wedge d \tau, \quad F^{A}=-\frac{1}{2} \mathcal{P}^{A} \sin \theta d \theta \wedge d \phi,
$$

whilst the scalar fields are

$$
z^{A}=-2 i \tilde{\phi}_{x} \beta q_{A+4} \sqrt{\frac{q_{0}}{q_{(1)}^{3}}}, \quad A=1,2,3,
$$

where $q_{5}=q_{(1)} \propto q_{6} \propto q_{7}$. For this model, the instanton solution is described (once the ratios of the scalar fields within the 3 by 3 block have been fixed) by the independent charges $\mathcal{Q}_{0}, \mathcal{P}^{(1)}$ and the 4 free parameters $B_{0}, B^{(1)}, h_{0}$ and $h^{(1)}$. The analysis of the conditions 
required for a regular black hole solution follow from the previous discussion and leads to the condition

$$
B_{0}=B^{(1)}=c .
$$

The Hamiltonian constraint (4.43) is then automatically satisfied. Asymptotic flatness leads to the further condition

$$
\left(-\frac{\mathcal{Q}_{0}}{c} \sinh \left(c \frac{h_{0}}{\mathcal{Q}_{0}}\right)\right)^{\frac{1}{2}}\left(\frac{\mathcal{P}^{(1)}}{c} \sinh \left(c \frac{h^{(1)}}{\mathcal{P}^{(1)}}\right)\right)^{\frac{3}{2}}=\beta
$$

on the integration constants $h_{0}, h^{(1)}$.

Finally, we can rewrite the four-dimensional metric (5.35) in terms of harmonic functions and the isotropic radial coordinate, $\rho$, as

$$
d s_{4}^{2}=-\frac{W}{\mathcal{H}_{0}^{\frac{1}{2}} \mathcal{H}^{(1) \frac{3}{2}}} d t^{2}+\mathcal{H}_{0}^{\frac{1}{2}} \mathcal{H}^{(1) \frac{3}{2}}\left(\frac{d \rho^{2}}{W}+\rho^{2} d \Omega_{(2)}^{2}\right),
$$

where the harmonic functions $W, \mathcal{H}_{0}$ and $\mathcal{H}^{(1)}$ are given by

$$
\begin{aligned}
W & =1-\frac{2 c}{\rho}=e^{-2 c \tau}, \\
\mathcal{H}_{0} & =-\beta^{\frac{1}{2}}\left[\frac{\mathcal{Q}_{0}}{c} \sinh \left(c \frac{h_{0}}{\mathcal{Q}_{0}}\right)+\mathcal{Q}_{0} e^{-c \frac{h_{0}}{\mathcal{Q}_{0}}} \frac{1}{\rho}\right] \\
& =-\beta^{\frac{1}{2}}\left[\frac{1}{2 c} \mathcal{Q}_{0} e^{c \frac{h_{0}}{\mathcal{Q}_{0}}}-\frac{1}{2 c} \mathcal{Q}_{0} e^{-c \frac{h_{0}}{\mathcal{Q}_{0}}} e^{-2 c \tau}\right], \\
\mathcal{H}^{(1)} & =\beta^{\frac{1}{2}}\left[\frac{\mathcal{P}^{(1)}}{c} \sinh \left(c \frac{h^{(1)}}{\mathcal{P}^{(1)}}\right)+\mathcal{P}^{(1)} e^{-c \frac{h^{(1)}}{\mathcal{P}^{(1)}}} \frac{1}{\rho}\right] \\
& =\beta^{\frac{1}{2}}\left[\frac{1}{2 c} \mathcal{P}^{(1)} e^{c \frac{h^{(1)}}{\mathcal{P}^{(1)}}}-\frac{1}{2 c} \mathcal{P}^{(1)} e^{-c \frac{h^{(1)}}{\mathcal{P}^{(1)}}} e^{-2 c \tau}\right] .
\end{aligned}
$$

The four-dimensional scalar fields are given by

$$
z^{A}=-2 i \tilde{\phi}_{x} \beta \xi_{4+A} \mathcal{H}^{(1)} \sqrt{\frac{\mathcal{H}_{0}}{\mathcal{H}^{(1)^{3}}}}, \quad A=1,2,3,
$$

where $\xi_{5}=1, \xi_{6}=\frac{\mathcal{P}^{2}}{\mathcal{P}^{1}}, \xi_{7}=\frac{\mathcal{P}^{3}}{\mathcal{P}_{1}}$, and we therefore have $z^{1} \propto z^{2} \propto z^{3}$.

\section{Black holes and first order equations}

For diagonal models, the general solution for spherically symmetric and purely imaginary field configurations (4.13) satisfies the $n+1$ first order equations

$$
\dot{q}_{\alpha}=\sqrt{\left(B_{\alpha} q_{\alpha}\right)^{2}+K_{\alpha}^{2}}, \quad \alpha=0, n+2, \ldots, 2 n+1 .
$$

Aside from the charges $K_{\alpha}$, these first order equations contain $n+1$ free parameters $B_{\alpha}$, which indicate that they have been obtained via integration from second order equations 
(the equations of motion (4.12)), and are therefore not unique. There are various different ways one may package the equations (6.1), for example one may write the r.h.s. in terms of $q^{\alpha}$ coordinates as

$$
\dot{q}_{\alpha}=\frac{\sqrt{B_{\alpha}^{\prime 2}+\left(K_{\alpha} q^{\alpha}\right)^{2}}}{q^{\alpha}},
$$

where the $B_{\alpha}^{\prime}$ are proportional ${ }^{14}$ to the constants $B_{\alpha}$. One may integrate the r.h.s. to obtain gradient-flow equations $\dot{q}_{\alpha}=\frac{\partial}{\partial q^{\alpha}} \mathcal{W}$, where

$$
4 \mathcal{W}=\sum_{\alpha}\left[\sqrt{B_{\alpha}^{\prime 2}+\left(K_{\alpha} q^{\alpha}\right)^{2}}+\frac{B_{\alpha}^{\prime}}{2} \log \left(\frac{\sqrt{B_{\alpha}^{\prime 2}+\left(K_{\alpha} q^{\alpha}\right)^{2}}-B_{\alpha}^{\prime}}{\sqrt{B_{\alpha}^{\prime 2}+\left(K_{\alpha} q^{\alpha}\right)^{2}}+B_{\alpha}^{\prime}}\right)\right] .
$$

A similar expression has been previously found for black hole solutions the STU model [56], which in our case corresponds to the specific choice $\lambda=1, n=3$. It is worth emphasising that the above gradient flow equations are valid for all solutions to the equations of motion, not just black holes, and that they depend on $n+1$ free parameters $\mathcal{W}=\mathcal{W}\left(B_{\alpha}^{\prime}\right)$. Therefore the existence of gradient flow equations does not mean that the solution satisfies a unique set of first order equations.

The situation is different for black hole solutions. We have shown that non-extremal black hole solutions are characterised by the requirement that $B_{\alpha}=c$ for all $\alpha$. In this case (6.1) reads

$$
\dot{q}_{\alpha}=\sqrt{c^{2} q_{\alpha}^{2}+K_{\alpha}^{2}} .
$$

Aside from the charges, these first-order equations contain just one free parameter: the non-extremality parameter $c$. In other words, we find that black hole solutions do satisfy a unique set of $n+1$ first order equations that depend only on the charges and nonextremality parameter. In this sense, black hole solutions are characterised by a reduction of the second order equations of motion to first order equations without increasing the number of equations. A similar conclusion was also found in our previous investigation into five-dimensional supergravity coupled to vector multiplets [20]. Counting the number of integration constants, we find that black hole solutions of the scalar fields $q_{\alpha}$ (or equivalently the complex scalar fields $z^{A}$, which are purely imaginary, plus the KK-scalar $e^{\phi}$ ) contain just $n+1$ integration constants, compared to $2 n+2$ that are present in the general solution of the equations of motion. For block-diagonal models the situation is entirely analogous, though in this case we only obtain as many first order equations as there are blocks in the metric.

\section{Conclusion and outlook}

In this paper we have continued to develop an approach to non-extremal solutions in $\mathcal{N}=2$ supergravity that is based on the real formulation of special geometry, dimensional reduction over time, and directly solving the second order field equations. Building upon [22] we have shown that non-extremal solutions with one or more non-constant scalar fields can be obtained for a large class of models by imposing conditions which lead to a block

\footnotetext{
${ }^{14}$ The exact relations are $B_{0}^{\prime}=\frac{\lambda}{2(\lambda+1)} B_{0}$ and $B_{A+(n+1)}^{\prime}=\frac{\lambda+2}{2 n(\lambda+1)} B_{A+(n+1)}$.
} 
decomposition of the equations of motion. Given our ability to a find at least one explicit non-trivial solution for each block, we can thus obtain explicit solutions, which for the specific conditions we imposed are given in terms of harmonic functions, as in (5.19), (5.21). Our method does not rely on group theoretical methods, and thus is not restricted to homogeneous spaces, nor does it rely on first order flow equations, and thus allows one to obtain solutions, and for some models the general solution, to the full second order equations of motion.

While we worked with ungauged supergravity and used the specific assumptions of spherical symmetry and purely imaginary scalar field configurations, it is clear that the method can be adapted to various other types of solutions, such as rotating black holes and black branes, in ungauged supergravity, gauged supergravity, and, more general EinsteinVector-Scalar theories with suitable conditions imposed on the couplings. We remark that various features which we can derive and understand systematically within our formalism have been observed and commented on in the literature for a variety of models and types of solutions. For example, the ansatz for non-extremal solutions which was recently outlined in [17] relies on various elements that we have seen at work in the present paper.

One observation commonly shared in the literature is that at least some non-extremal solutions preserve features of BPS solutions. In our work this is manifest when expressing the solutions in terms of harmonic functions, as in (5.19), (5.21): the line element and gauge fields are modified universally by the additional harmonic function $W$, while the scalar solution has exactly the same form as in the BPS case. What changes compared to the BPS case are the expressions for the constants within the harmonic functions, which now depend on the non-extremality parameter $c$.

Another universal observation is that (at least some and maybe all) non-extremal black hole solutions satisfy unique first order equations. In our approach this is not an ansatz or a condition that we impose, but follows when we select from the general solution of the second order equations the subset that describes regular black hole solutions. This reduces the number of integration constants by one half, and as a result we can demonstrate that the general black hole solution satisfies a unique set of first order equations. For BPS and more generally extremal solutions the same phenomenon is know to result from the fixed point behaviour implied by the black hole attractor mechanism. Since there is no fixed point behaviour for non-extremal solutions, it is at first surprising that some, and possibly all non-extremal solutions satisfy first order equations. But, as already discussed in $[18,19,57]$ some features commonly associated with the attractor mechanism persist for non-extremal solutions.

In fact the synonym 'stabilisation equations' for the BPS attractor equations reflects that obtaining BPS solutions with regular horizons requires to impose conditions on the scalar field to 'stabilise' them on the horizon. For BPS solutions this is realised by the asymptotic restoration of full supersymmetry which makes the near horizon solution a supersymmetric ground state [58-60]. The difference between the extremal and non-extremal case is that the near horizon solution is a ground state, which forces the scalars to take fixed point values which are exclusively determined by the electric and magnetic charges. In the non-extremal case the scalar flow reaches the horizon before reaching a fixed point, 
and the horizon values of the scalar are not determined by the gauge charges. But they are still not independent integration constants, as they would be if we considered the full second order scalar equations without regularity conditions at the horizon. Instead they are determined by other integration constants, namely the gauge charges together with the asymptotic values of the scalars at infinity. It is therefore not unreasonable to expect that the scalar flow between infinity and horizon is always governed by first order equations which result from deforming the first order equations valid for the extremal case. While we have demonstrated this here for a large class of models, it remains to investigate whether this is true in general.

In the case that the target manifold is a Riemannian symmetric space it has previously been observed that the coefficient of the leading order term in the $1 / \rho$ expansion of the scalar fields, referred to as the scalar charge, is not an independent parameter for black holes solutions [26]. In this paper we have constructed full analytic solutions to the equations of motion, and therefore the reduction in the number of free parameters in the solution is a stronger statement, even when the target manifold is a symmetric space. In fact, by considering the $1 / \rho$ expansion one automatically finds that the scalar charge is not an independent parameter, regardless of whether or not the target manifold is symmetric.

While we have only obtained the general solution to the second order equations of motion for diagonal models, these form a large class of models with, up to two exceptions, non-homogeneous target spaces. Moreover we saw that the observed pattern persisted for block-diagonal models, where we could obtain a subset of solutions to the second order equations and still observe that regularity at the horizon reduces the number of integration constants by one half. We do not see any reason why these systematic features should only apply to models where we can solve the equations of motion explicitly, and expect that they are generic.

Another universal feature, which for example has also been mentioned recently in [17] is that our ability to find explicit non-extremal solutions results from a symmetry of the equations of motion. In our case the relevant symmetry only comes into existence after consistently truncating out half of the scalars by the PI conditions. The resulting block decomposition of the Hessian metric implies an invariance of the equations of motion under a field rotation matrix, which was discussed in detail in [22] for the special case of prepotentials with $\lambda=1$. We note that this symmetry can always be used for both generating non-BPS extremal solutions from BPS solutions (as done in [22]), and to obtain non-extremal solutions (as done in the present paper). This is complementary to the observation that BPS and non-BPS extremal solutions can be 'unified' through obtaining them both as limits of non-extremal solutions [56].

There are various directions to be explored in the future. As already mentioned the formalism developed here can be extended and adapted to gauged supergravity and other types of solutions. It would be interesting to find situations where a block decomposition is possible but the solutions for individual blocks are not harmonic functions. For example, some multi-centered extremal solutions found for symmetric target spaces contain nonharmonic functions [61], and so-called unconventional solutions involving anharmonic terms were constructed in [62]. 
One limitation of the PI condition is that eliminates half of the charges and at least half of the independent scalar fields. This was necessary in order to obtain a block decomposition and to get rid of the terms in the second and third line of (2.14). However for some models with symmetric target spaces solutions with all charges turned on are known, and for the $S T U$ model the general charged rotating solution (including NUT charge) was found in [63]. It would be interesting to obtain solutions with more charges turned on for non-symmetric and in fact non-homogeneous target spaces.

While in this paper we have focused on obtaining explicit solutions in closed form, there is a complementary, more geometrical approach about which we will report elsewhere $[25,64]$. The target manifold of the three-dimensional Euclidean theory is a paraquaternionic Kähler manifold (as is proved in generality in [24, 25]), and the construction of solutions is facilitated by constructing harmonic maps onto totally geodesic submanifolds. The submanifold corresponding to static, purely imaginary field configurations is in fact a para-Kähler submanifold, which contains the 'black string submanifold' already identified in [21]. Further para-Kähler submanifolds can be constructed systematically [57, 64]. One interesting question for the future is to relate this approach to the group-theoretical approach which works so well if the target space is a symmetric space. This will hopefully lead to further insights which will allow us to obtain a systematic understanding of non-extremal (and also of extremal) solutions for generic $\mathcal{N}=2$ string compactifications.

In this paper we have mostly restricted our attention to those solutions which are regular four-dimensional black holes. Within the full class of solutions we constructed, there should be interesting subclasses corresponding to three-dimensional instanton solutions with finite action, and to black hole solutions that are only regular when lifting to dimensions higher than four. We leave this investigation to future work.

\section{Acknowledgments}

The work of T.M. is supported in part by STFC grant ST/G00062X/1. The work of D.E. is supported by STFC studentship ST/K502145/1. The work of O.V. is supported by the German Science Foundation (DFG) under the Collaborative Research Center (SFB) 676 "Particles, Strings and the Early Universe." T.M. thanks the Department of Mathematics at the University of Hamburg for hospitality and support during various stages of this work.

\section{A Hessian geometry}

\section{A.1 The Hesse potential $H$}

In this appendix we collect or prove certain identities for Hessian metrics which we use in the paper or find generally noteworthy.

In terms of affine coordinates $q^{a}$ a Hessian metric $H_{a b}$ is given by the second derivatives of a real valued function, the Hesse potential $H$

$$
H_{a b}=\frac{\partial^{2} H}{\partial q^{a} \partial q^{b}}
$$


The coordinate-independent definition requires the existence of a flat, torsion free connection $\nabla$, such that the rank three tensor $\nabla g$, where $g$ is the metric, is totally symmetric [55]. The affine coordinates $q^{a}$ are then defined by $\nabla d q^{a}=0$.

Affine special Kähler (ASK) manifolds are simultaneously Kähler and Hessian. ${ }^{15}$ One can choose special real coordinates $q^{a}$ which are affine coordinates with respect to the Hessian structure and simultaneously Darboux coordinates, that is the Kähler form is constant in these coordinates [43]:

$$
\omega=\Omega_{a b} d q^{a} \wedge d q^{b}, \quad\left(\Omega_{a b}\right)=\left(\begin{array}{cc}
0 & \mathbb{1} \\
-\mathbb{1} & 0
\end{array}\right) .
$$

The associated complex structure is

$$
J_{c}^{a}=-\frac{1}{2} \Omega^{a b} H_{b c} .
$$

It is useful to note the equivalent relation

$$
H_{a b} \Omega^{b c} H_{c d}=-4 \Omega_{a b}
$$

In $\mathcal{N}=2$ supergravity the Hesse potential $H$ is homogeneous of degree 2, which implies the relations

$$
q^{a} H_{a}=2 H, \quad q^{a} H_{a b}=H_{b}, \quad q^{a} H_{a b c}=0,
$$

where $H_{a}=\frac{\partial H}{\partial q^{a}}$, etc. This implies that the affine special Kähler manifold is conical, see [27] for a coordinate-free definition. While in general the Hesse potential is only unique up to affine transformations, preserving homogeneity restricts this to linear transformations, and chooses affine coordinates which are adapted to the conical structure. Moreover, for special real coordinates one also imposes that the Kähler form is invariant, which further restricts the linear transformations to be symplectic. In the following it is understood that we use special coordinates which are adapted to the conical structure.

Affine special Kähler manifolds come in fact equipped with a one-parameter family of special connections $\nabla$, each with its own system of special real coordinates [44]. In particular, dual special real coordinates are defined by

$$
q_{a}^{\prime}=H_{a}=\frac{\partial H}{\partial q^{a}} .
$$

Since $H$ is homogeneous of degree 2, the special coordinates and dual special coordinates are related by

$$
q_{a}^{\prime}=H_{a b} q^{b} \Leftrightarrow q^{q}=H^{a b} q_{b}^{\prime},
$$

where $H^{a b}$ denotes the inverse of the Hessian metric $H_{a b}$. Since $q_{a}^{\prime}$ are special real coordinates, and the metric is

$$
g=H_{a b} d q^{a} d q^{b}=H^{a b} d q_{a}^{\prime} d q_{b}^{\prime},
$$

\footnotetext{
${ }^{15}$ We are using the formulation of special geometry developed in [44]. The relevant facts are reviewed in $[22]$.
} 
there exists a Hesse potential $H^{\prime}\left(q_{a}^{\prime}\right)$ for the inverse metric $H^{a b}$ :

$$
H^{a b}=\frac{\partial^{2} H^{\prime}}{\partial q_{a}^{\prime} \partial q_{b}^{\prime}} .
$$

We now show that corresponding Hesse potential $H^{\prime}\left(q^{\prime}\right)$ is given by transforming $H(q)$ with the diffeomorphism $q^{a} \mapsto q_{a}^{\prime}$, that is $H^{\prime}\left(q^{\prime}\right)=H\left(q\left(q^{\prime}\right)\right)$. Note that the diffeomorphism $q^{a} \mapsto q_{a}^{\prime}$ is in general non-linear (unless $H_{a b}$ is constant), and therefore does not preserve the affine structure determined by a given fixed special connection $\nabla$. As already mentioned $q^{a}$ and $q_{a}^{\prime}$ are special real coordinates with respect to two different affine structures, and in particular need not be related by a symplectic transformation.

Begin proof. First note that

$$
\frac{\partial q_{a}^{\prime}}{\partial q^{b}}=H_{a b}, \quad \frac{\partial q^{a}}{\partial q_{b}^{\prime}}=H^{a b} .
$$

Since $\partial q_{a}^{\prime} / \partial q^{b}$ is the Jacobian of the transformation $q^{a} \mapsto q_{a}^{\prime}$, it is clear that the metric coefficients with respect to the coordinates $q_{a}^{\prime}$ are the inverse $H^{a b}$ of the metric coefficients $H_{a b}$ with respect to $q^{a}$. Since $q_{a}^{\prime}$ are special real coordinates, there exists as a Hesse potential $H^{\prime}\left(q^{\prime}\right)$

$$
H^{a b}=\frac{\partial^{2} H^{\prime}}{\partial q_{a}^{\prime} \partial q_{b}^{\prime}},
$$

which is homogeneous of degree two. Our claim is that $H^{\prime}\left(q^{\prime}\right)$ is related to $H(q)$ by $H^{\prime}\left(q^{\prime}\right)=H\left(q\left(q^{\prime}\right)\right)$. Since this is equivalent to $H(q)=H^{\prime}\left(q^{\prime}(q)\right)$, we can prove instead that $H^{\prime}\left(q^{\prime}(q)\right)$ is a Hesse potential for $H_{a b}$, i.e.

$$
H_{a b}=\frac{\partial^{2} H^{\prime}}{\partial q^{a} \partial q^{b}}
$$

Using the chain rule, we compute

$$
\frac{\partial^{s} H^{\prime}}{\partial q^{a} \partial q^{b}}=\frac{\partial^{2} H^{\prime}}{\partial q_{c}^{\prime} \partial q_{d}^{\prime}} \frac{\partial q_{c}^{\prime}}{\partial q^{a}} \frac{\partial q_{d}^{\prime}}{\partial q^{b}}+\frac{\partial H^{\prime}}{\partial q_{c}^{\prime}} \frac{\partial^{2} q_{c}^{\prime}}{\partial q^{a} \partial q^{b}}=H^{c d} H_{c a} H_{d b}+\frac{\partial H^{\prime}}{\partial q_{c}^{\prime}} H_{a b c} .
$$

Then it remains to show that the second term is zero. We note that

$$
\frac{\partial^{2} H^{\prime}}{\partial q_{a}^{\prime} \partial q_{b}^{\prime}}=H^{a b}=\frac{\partial q^{a}}{\partial q_{b}^{\prime}},
$$

which can be integrated to

$$
\frac{\partial H^{\prime}}{\partial q_{a}^{\prime}}=q^{a} .
$$

Note that there is no integration constant since $H^{\prime}$ is homogeneous of degree two. Using homogeneity we find

$$
\frac{\partial H^{\prime}}{\partial q_{c}^{\prime}} H_{a b c}=q^{c} H_{a b c}=0,
$$

so that

$$
\frac{\partial^{2} H^{\prime}}{\partial q^{a} \partial q^{b}}=H^{c d} H_{c a} H_{d b}=H_{a b} .
$$




\section{A.2 The Hesse potential $\tilde{H}$}

Given a Hesse potential $H(q)$ which is homogeneous of degree two, ${ }^{16}$ we can define a new Hesse potential by

$$
\tilde{H}=C \log H,
$$

where $C$ is a constant. In the main part of the paper, we have to choose $C=-\frac{1}{2}$ in order for (2.15) to hold true. Note that if we replace $H$ by $\alpha H$ in (A.1), where $\alpha \in \mathbb{R} \backslash\{0\}$, all derivatives remain unchanged and, since it is derivatives of $\tilde{H}$ that appear in the equations of motion, we are free to make such a change. A constant $\alpha<0$ is for example required if $H<0$ in order that the argument of the logarithm is positive. In the main part of the paper we choose $\alpha=-2$, as in (2.15), because this is convenient when imposing the D-gauge condition (2.12).

Returning to our analysis of the general formula (A.1), we see that while $\tilde{H}$ is not a homogeneous function, its $n$-th derivative is homogeneous of degree $-n$ for $n \geq 1$. Therefore the Hessian metric defined by

$$
\tilde{H}_{a b}=\frac{\partial^{2} \tilde{H}}{\partial q^{a} \partial q^{b}}
$$

has metric coefficients which are homogeneous of degree -2 , while the metric tensor $\tilde{g}=$ $\tilde{H}_{a b} d q^{a} d q^{b}$ is homogeneous of degree 0 . The metric coefficients can be expressed in terms of $H$ by

$$
\tilde{H}_{a b}=C \frac{H_{a b} H-H_{a} H_{b}}{H^{2}} .
$$

Using homogeneity, it is straightforward to verify that the inverse metric has coefficients

$$
\tilde{H}^{a b}=C^{-1}\left(H H^{a b}-q^{a} q^{b}\right) .
$$

We define dual coordinates with respect to $\tilde{H}$ by

$$
q_{a}:=\tilde{H}_{a}:=\frac{\partial \tilde{H}}{\partial q^{a}}=C \frac{H_{a}}{H}=C \frac{q_{a}^{\prime}}{H} .
$$

Then

$$
\tilde{H}_{a b}=\frac{\partial q_{a}}{\partial q^{b}} \Rightarrow \tilde{H}^{a b}=\frac{\partial q^{a}}{\partial q_{b}} .
$$

Since $\tilde{H}_{a}$ is homogeneous of degree -1 :

$$
\tilde{H}_{a b} q^{b}=-\tilde{H}_{a}=-q_{a} \Rightarrow q^{a}=-\tilde{H}^{a b} q_{b} .
$$

Due to the additional minus sign, the coordinates $q^{a}$ and $q_{a}$ are not simply related by 'lowering the index' using the metric $\tilde{H}_{a b}$. Since coordinates are functions, and not vector

\footnotetext{
${ }^{16}$ Generalising the following discussion to the case where $H(q)$ has an arbitrary degree of homogeneity is straightforward and only changes some numerical coefficients in the formulae given in this section. In five dimensions one can consider non-supersymmetric theories based on 'generalised special real geometry', and it turns out that black brane solutions can be constructed by the same methods as used in supergravity [18, 20, 21]. Here we focus on the case of degree two for concreteness, and because it is the case we consider in this paper.
} 
fields on the underlying manifold $M$, there is nothing wrong with this relation. We do of course observe the standard tensorial behaviour when considering the action of the metric on tensors, such as tangent vectors to curves

$$
\dot{q}^{a}=\tilde{H}^{a b} \dot{q}_{b}
$$

partial derivatives ${ }^{17} \frac{\partial}{\partial q^{q}}$ and differentials like $d q^{a}$.

We can define a dual Hesse potential $\tilde{H}^{\prime}\left(q_{b}\right):=\tilde{H}\left(q^{a}\left(q_{b}\right)\right)$. Then

$$
\frac{\partial \tilde{H}^{\prime}}{\partial q_{a}}=\frac{\partial \tilde{H}}{\partial q^{b}} \frac{\partial q^{b}}{\partial q_{a}}=\frac{\partial \tilde{H}}{\partial q^{b}} \tilde{H}^{b a}
$$

which implies that

$$
q^{a}=-\tilde{H}^{a b} q_{b}=-\tilde{H}^{a b} \frac{\partial \tilde{H}}{\partial q^{b}}=-\frac{\partial \tilde{H}^{\prime}}{\partial q_{a}} .
$$

Therefore $-\tilde{H}^{\prime}$ is a Hesse potential for the inverse $\tilde{H}^{a b}$ of $\tilde{H}_{a b}$ :

$$
\tilde{H}^{a b}=\frac{\partial q^{a}}{\partial q_{b}}=\frac{\partial^{2}\left(-\tilde{H}^{\prime}\right)}{\partial q_{a} \partial q_{b}}
$$

We add some useful relations between the two types of dual coordinates, $q_{a}^{\prime}=H_{a}$ and $q_{a}=\tilde{H}_{a}$. From the definition (A.3) of $q_{a}$ we derive

$$
\frac{\partial q_{a}}{\partial q_{b}^{\prime}}=C \frac{\delta_{a}^{b} H-q_{a}^{\prime} q^{b}}{H^{2}} \Rightarrow \frac{\partial q_{a}^{\prime}}{\partial q_{b}}=C^{-1}\left(\delta_{a}^{b} H-q_{a}^{\prime} q^{b}\right) .
$$

Using this, one can derive the relations (A.6), (A.2) and (A.7) directly by differentiating the dual Hesse potential $\tilde{H}^{\prime}\left(q_{a}\right)=C \log H^{\prime}\left(q^{\prime}\left(q_{a}\right)\right.$ :

$$
\frac{\partial \tilde{H}^{\prime}}{\partial q_{a}}=-q^{a}, \quad \frac{\partial^{2} \tilde{H}^{\prime}}{\partial q_{a} \partial q_{b}}=-C^{-1}\left(H^{a b} H-q^{a} q^{b}\right)=-\tilde{H}^{a b} .
$$

In the paper we compute $\tilde{H}^{a b}$ by (A.7) with $\tilde{H}^{\prime}=C \log H^{\prime \prime}$ where $H^{\prime \prime}\left(q_{b}\right)=H\left(q^{a}\left(q_{b}\right)\right)$ :

$$
\tilde{H}^{a b}=C\left(\frac{1}{H^{\prime \prime}} \frac{\partial^{2} H^{\prime \prime}}{\partial q_{a} \partial q_{b}}-\frac{1}{H^{2}} \frac{\partial H^{\prime \prime}}{\partial q_{a}} \frac{\partial H^{\prime \prime}}{\partial q_{b}}\right) .
$$

Using the Jacobian $\frac{\partial q_{a}}{\partial q_{b}^{\prime}}$ given above it is straightforward to check that this is related to (A.2) by a change of variables.

For notational simplicity, we have usually dropped the primes on $\tilde{H}^{\prime}, H^{\prime}, H^{\prime \prime}$ in the main part of the paper, whenever it is clear from context which variables the function depends on.

\footnotetext{
${ }^{17}$ Since $q^{a}$ are affine coordinate with respect to the flat, torsion-free connection $\nabla$ defining the Hessian structure, partial derivatives coincide with covariant derivatives in this coordinate system, and hence define a covariant object.
} 


\section{B Spherically symmetric metrics}

Here we will review material from [65, 66].

A spacetime is said to be spherically symmetric if the isometry group contains a subgroup isomorphic to $\mathrm{SO}(3)$, and the orbits of this subgroup are two-spheres. We may therefore interpret $\mathrm{SO}(3)$ transformations as rotations.

The spacetime metric induces a metric on each orbit two-sphere. Since the orbits are two-dimensional submanifolds, and a three-dimensional isometry group is the maximum possible, ${ }^{18} 3=2(2+1) / 2$, the curvature of the two-spheres must be constant. The metric on the orbit two-spheres must therefore be proportional to the metric on the unit twosphere. By theorem 3 of [67] at each point the orbit two-spheres are orthogonal to a twodimensional timelike submanifold, which we parametrise by $(r, t)$. The spacetime metric therefore decomposes into two blocks

$$
d s^{2}=\left[-A^{2}(r, t) d t^{2}+B(r, t) d t d r+C^{2}(r, t) d r^{2}\right]+D^{2}(r, t)\left(d \theta^{2}+\sin ^{2} \theta d \varphi^{2}\right) .
$$

In these coordinates a basis of $\mathrm{SO}(3)$ rotations is given by

$$
\begin{aligned}
& \eta_{1}=-\cos \varphi \partial_{\theta}+\cot \theta \sin \varphi \partial_{\varphi} \\
& \eta_{2}=\sin \varphi \partial_{\theta}+\cot \theta \cos \varphi \partial_{\varphi} \\
& \eta_{3}=\partial_{\varphi}
\end{aligned}
$$

\section{B.1 Stationary and spherically symmetric}

A spacetime is said to be stationary if the isometry group contains a one-parameter subgroup with orbits given by timelike curves, which we parametrise by $t$. This is equivalent to the existence of a timelike Killing vector field $\xi=\partial_{t}$, which we assume to be unique.

Consider a spacetime that is both stationary and spherically symmetric. Due to the uniqueness of $\xi$ it is orthogonal to the $\mathrm{SO}(3)$ orbit two-spheres [66]. This means that the decomposition of the metric according to (B.1) is compatible with the choice of $t$ as a timelike coordinate, and since $t$ parametrises an isometry the components of the metric must be independent of this parameter

$$
d s^{2}=\left[-A(r)^{2} d t^{2}+B(r) d t d r+C(r)^{2} d r^{2}\right]+D^{2}(r)\left(d \theta^{2}+\sin ^{2} \theta d \varphi^{2}\right) .
$$

Let us investigate the function $D$ further. Setting $D$ to be constant, i.e. $\nabla_{\mu} D=0$ (= $\left.\partial_{r} D\right)$, is inconsistent with the equations of motion for either a vacuum solution or a static perfect fluid solution [65], and is therefore not considered physical. We therefore assume $\nabla_{\mu} D \neq 0$, and we may use the function $D$ as a spacetime coordinate

$$
\tilde{r}:=D(r), \quad \frac{d \tilde{r}}{d r}=\partial_{r} D \neq 0,
$$

\footnotetext{
${ }^{18}$ The data needed to describe a Killing vector at a point are $\xi^{\mu}$ and $\nabla_{[\mu} \xi_{\nu]}$. This is because all higher derivatives are determined by the Riemann curvature tensor through

$$
\nabla_{\mu} \nabla_{\nu} \xi_{\rho}=R_{\mu \nu \rho}{ }^{\sigma} \xi_{\sigma} .
$$

We therefore count $d$ independent degrees of freedom from $\xi^{\mu}$, and $(d-1) / 2$ from $\nabla_{[\mu} \xi_{\nu]}$. The maximum number of Killing vectors is therefore $d(d+1) / 2$.
} 
in which case the metric takes the form

$$
d s^{2}=\left[-\tilde{A}(\tilde{r})^{2} d t^{2}+\tilde{B}(\tilde{r}) d t d \tilde{r}+\tilde{C}(\tilde{r})^{2} d \tilde{r}^{2}\right]+\tilde{r}^{2}\left(d \theta^{2}+\sin ^{2} \theta d \varphi^{2}\right) .
$$

We shall now review the argument that a stationary and spherically symmetric spacetime is necessarily static. First note that

$$
\nabla_{\xi} \tilde{r}=\nabla_{\xi} D(r)=\partial_{t} D(r)=0
$$

From this expression we can see that the covectors $g(\xi, \cdot)$ and $\nabla \tilde{r}=d \tilde{r}$ are orthogonal, which means that the corresponding vectors

$$
\xi=\partial_{t} \quad \text { and } \quad \psi=\left(\nabla^{\mu} \tilde{r}\right) \partial_{\mu}
$$

are orthogonal since they are obtained by raising indices using the metric. Using the decomposition of the metric we can write $\psi$ as

$$
\begin{aligned}
\psi & =g^{\tilde{r} t} \frac{\partial}{\partial t}+g^{\tilde{r} \tilde{r}} \frac{\partial}{\partial \tilde{r}} \\
& =\frac{-1}{\tilde{A}^{2} \tilde{C}^{2}+\frac{1}{4} \tilde{B}^{2}}\left(-\frac{1}{2} \tilde{B} \frac{\partial}{\partial t}-\tilde{A}^{2} \frac{\partial}{\partial \tilde{r}}\right) .
\end{aligned}
$$

Since $\xi$ and $\psi$ are orthogonal it follows that $\tilde{B}=0$. It is also clear that $\psi, \partial_{\theta}, \partial_{\varphi}$ commute, and therefore define a three-dimensional integrable distribution. Let us denote the corresponding hypersurface by $\Sigma$, which may be locally parametrised by $(\tilde{r}, \theta, \varphi)$. Since $\Sigma$ is orthogonal to the timelike Killing vector $\xi$ we have proved that the spacetime is static. The metric takes the form

$$
d s^{2}=-\tilde{A}(\tilde{r})^{2} d t^{2}+\tilde{C}(\tilde{r})^{2} d r^{2}+\tilde{r}^{2}\left(d \theta^{2}+\sin ^{2} \theta d \varphi^{2}\right) .
$$

We end by making the coordinate transformation $\tau=\int \frac{\tilde{C}(\tilde{r})}{\tilde{r}^{2} \tilde{A}(\tilde{r})} d \tilde{r}$, in which case the metric may be written as

$$
d s^{2}=-\tilde{A}(\tau)^{2} d t^{2}+\tilde{A}(\tau)^{-2}\left[e^{4 \mathcal{A}(\tau)} d \tau^{2}+e^{2 \mathcal{A}(\tau)}\left(d \theta^{2}+\sin ^{2} \theta d \varphi^{2}\right)\right],
$$

where $e^{\mathcal{A}(\tau)}:=\tilde{r} \tilde{A}(\tilde{r})$. The advantage of this parametrisation is that $\Delta f=\frac{d^{2}}{d \tau^{2}} f$ for all functions $f=f(\tau)$ that only depend on the radial coordinate, which leads to simplifications in the equations of motion. In particular $\tau$ provides an affine parametrisation of the geodesic curve $\left(q^{a}(\tau), \hat{q}^{a}(\tau)\right)$ on the scalar manifold corresponding to the solution of the scalar field equations.

Open Access. This article is distributed under the terms of the Creative Commons Attribution License (CC-BY 4.0), which permits any use, distribution and reproduction in any medium, provided the original author(s) and source are credited. 


\section{References}

[1] E. Bergshoeff, W. Chemissany, A. Ploegh, M. Trigiante and T. Van Riet, Generating Geodesic Flows and Supergravity Solutions, Nucl. Phys. B 812 (2009) 343 [arXiv:0806.2310] [INSPIRE].

[2] W. Chemissany, J. Rosseel, M. Trigiante and T. Van Riet, The full integration of black hole solutions to symmetric supergravity theories, Nucl. Phys. B 830 (2010) 391 [arXiv: 0903.2777] [INSPIRE].

[3] W. Chemissany et al., Black holes in supergravity and integrability, JHEP 09 (2010) 080 [arXiv: 1007.3209] [INSPIRE].

[4] M. Cvetič and D. Youm, Dyonic BPS saturated black holes of heterotic string on a six torus, Phys. Rev. D 53 (1996) 584 [hep-th/9507090] [INSPIRE].

[5] M. Cvetič and A.A. Tseytlin, Solitonic strings and BPS saturated dyonic black holes, Phys. Rev. D 53 (1996) 5619 [hep-th/9512031] [InSPIRE].

[6] M. Cvetič and D. Youm, General rotating five-dimensional black holes of toroidally compactified heterotic string, Nucl. Phys. B 476 (1996) 118 [hep-th/9603100] [INSPIRE].

[7] M. Cvetič and D. Youm, Entropy of nonextreme charged rotating black holes in string theory, Phys. Rev. D 54 (1996) 2612 [hep-th/9603147] [INSPIRE].

[8] H. Lü, C.N. Pope and J.F. Vazquez-Poritz, From AdS black holes to supersymmetric flux branes, Nucl. Phys. B 709 (2005) 47 [hep-th/0307001] [inSPIRE].

[9] C.M. Miller, K. Schalm and E.J. Weinberg, Nonextremal black holes are BPS, Phys. Rev. D 76 (2007) 044001 [hep-th/0612308] [InSPIRE].

[10] A. Ceresole and G. Dall'Agata, Flow Equations for Non-BPS Extremal Black Holes, JHEP 03 (2007) 110 [hep-th/0702088] [INSPIRE].

[11] L. Andrianopoli, R. D'Auria, E. Orazi and M. Trigiante, First order description of black holes in moduli space, JHEP 11 (2007) 032 [arXiv:0706.0712] [INSPIRE].

[12] B. Janssen, P. Smyth, T. Van Riet and B. Vercnocke, A first-order formalism for timelike and spacelike brane solutions, JHEP 04 (2008) 007 [arXiv:0712.2808] [INSPIRE].

[13] G.L. Cardoso and V. Grass, On five-dimensional non-extremal charged black holes and FRW cosmology, Nucl. Phys. B 803 (2008) 209 [arXiv:0803.2819] [INSPIRE].

[14] J. Perz, P. Smyth, T. Van Riet and B. Vercnocke, First-order flow equations for extremal and non-extremal black holes, JHEP 03 (2009) 150 [arXiv:0810.1528] [INSPIRE].

[15] J.B. Gutowski and W.A. Sabra, Five Dimensional Non-Supersymmetric Black Holes and Strings, JHEP 05 (2009) 092 [arXiv:0803.3189] [INSPIRE].

[16] S. Barisch, G. Lopes Cardoso, M. Haack, S. Nampuri and N.A. Obers, Nernst branes in gauged supergravity, JHEP 11 (2011) 090 [arXiv:1108.0296] [INSPIRE].

[17] K. Goldstein, S. Nampuri and Á. Véliz-Osorio, Heating up branes in gauged supergravity, JHEP 08 (2014) 151 [arXiv:1406.2937] [InSPIRE].

[18] T. Mohaupt and K. Waite, Instantons, black holes and harmonic functions, JHEP 10 (2009) 058 [arXiv: 0906.3451] [INSPIRE].

[19] T. Mohaupt and O. Vaughan, Non-extremal Black Holes, Harmonic Functions and Attractor Equations, Class. Quant. Grav. 27 (2010) 235008 [arXiv:1006.3439] [InSPIRE]. 
[20] T. Mohaupt and O. Vaughan, Non-extremal black holes from the generalised R-map, Springer Proc. Phys. 144 (2013) 233 [arXiv:1208.4302] [INSPIRE].

[21] P. Dempster and T. Mohaupt, Non-extremal and non-BPS extremal five-dimensional black strings from generalized special real geometry, Class. Quant. Grav. 31 (2014) 045019 [arXiv: 1310.5056] [INSPIRE].

[22] T. Mohaupt and O. Vaughan, The Hesse potential, the c-map and black hole solutions, JHEP 07 (2012) 163 [arXiv:1112.2876] [INSPIRE].

[23] V. Cortés, C. Mayer, T. Mohaupt and F. Saueressig, Special geometry of Euclidean supersymmetry. 1. Vector multiplets, JHEP 03 (2004) 028 [hep-th/0312001] [INSPIRE].

[24] O. Vaughan, The r-map, the c-map and black hole solutions, Ph.D. Thesis, University of Liverpool, Liverpool, U.K. (2012).

[25] V. Cortés, P. Dempster, T. Mohaupt and O. Vaughan, Special Geometry of Euclidean Supersymmetry IV: hypermultiplets and local c-maps, to appear.

[26] P. Breitenlohner, D. Maison and G.W. Gibbons, Four-Dimensional Black Holes from Kaluza-Klein Theories, Commun. Math. Phys. 120 (1988) 295 [INSPIRE].

[27] V. Cortés and T. Mohaupt, Special Geometry of Euclidean Supersymmetry III: The local r-map, instantons and black holes, JHEP 07 (2009) 066 [arXiv:0905. 2844] [INSPIRE].

[28] D. Klemm and O. Vaughan, Nonextremal black holes in gauged supergravity and the real formulation of special geometry, JHEP 01 (2013) 053 [arXiv:1207.2679] [INSPIRE].

[29] D. Klemm and O. Vaughan, Nonextremal black holes in gauged supergravity and the real formulation of special geometry II, Class. Quant. Grav. 30 (2013) 065003 [arXiv:1211.1618] [INSPIRE].

[30] T. Mohaupt and K. Waite, Euclidean Actions, Instantons, Solitons and Supersymmetry, J. Phys. A 44 (2011) 175403 [arXiv:1011.6301] [InSPIRE].

[31] B. de Wit, V. Kaplunovsky, J. Louis and D. Lüst, Perturbative couplings of vector multiplets in $N=2$ heterotic string vacua, Nucl. Phys. B 451 (1995) 53 [hep-th/9504006] [INSPIRE].

[32] J.A. Harvey and G.W. Moore, Algebras, BPS states and strings, Nucl. Phys. B 463 (1996) 315 [hep-th/9510182] [INSPIRE].

[33] J. Louis, J. Sonnenschein, S. Theisen and S. Yankielowicz, Nonperturbative properties of heterotic string vacua compactified on $K 3 \times T^{2}$, Nucl. Phys. B 480 (1996) 185 [hep-th/9606049] [INSPIRE].

[34] T. Mohaupt, Black hole entropy, special geometry and strings, Fortsch. Phys. 49 (2001) 3 [hep-th/0007195] [INSPIRE].

[35] B. de Wit and A. Van Proeyen, Special geometry, cubic polynomials and homogeneous quaternionic spaces, Commun. Math. Phys. 149 (1992) 307 [hep-th/9112027] [INSPIRE].

[36] A. Gnecchi, K. Hristov, D. Klemm, C. Toldo and O. Vaughan, Rotating black holes in 4d gauged supergravity, JHEP 01 (2014) 127 [arXiv:1311.1795] [INSPIRE].

[37] P. Meessen, T. Ortín, J. Perz and C.S. Shahbazi, H-FGK formalism for black-hole solutions of $N=2, D=4$ and $D=5$ supergravity, Phys. Lett. B 709 (2012) 260 [arXiv:1112.3332] [INSPIRE].

[38] S. Ferrara, G.W. Gibbons and R. Kallosh, Black holes and critical points in moduli space, Nucl. Phys. B 500 (1997) 75 [hep-th/9702103] [INSPIRE]. 
[39] P. Galli, T. Ortín, J. Perz and C.S. Shahbazi, Black hole solutions of $N=2, D=4$ supergravity with a quantum correction, in the H-FGK formalism, JHEP 04 (2013) 157 [arXiv: 1212.0303] [INSPIRE].

[40] P. Bueno, R. Davies and C.S. Shahbazi, Quantum Black Holes in Type-IIA String Theory, JHEP 01 (2013) 089 [arXiv:1210.2817] [INSPIRE].

[41] B. de Wit and A. Van Proeyen, Potentials and Symmetries of General Gauged N=2 Supergravity: Yang-Mills Models, Nucl. Phys. B 245 (1984) 89 [INSPIRE].

[42] D.Z. Freedman and A. Van Proeyen, Supergravity, Cambridge University Press, Cambridge, U.K. (2012).

[43] D.S. Freed, Special Kähler manifolds, Commun. Math. Phys. 203 (1999) 31 [hep-th/9712042] [INSPIRE].

[44] D.V. Alekseevsky, V. Cortés and C. Devchand, Special complex manifolds, J. Geom. Phys. 42 (2002) 85 [math/9910091] [INSPIRE].

[45] N.J. Hitchin, The Moduli space of complex Lagrangian submanifolds, Asian J. Math 3 (1999) 77 [math/9901069] [INSPIRE].

[46] G. Lopes Cardoso, B. de Wit, J. Kappeli and T. Mohaupt, Black hole partition functions and duality, JHEP 03 (2006) 074 [hep-th/0601108] [INSPIRE].

[47] G.L. Cardoso, B. de Wit and S. Mahapatra, BPS black holes, the Hesse potential and the topological string, JHEP 06 (2010) 052 [arXiv: 1003.1970] [INSPIRE].

[48] G.L. Cardoso, B. de Wit and S. Mahapatra, Non-holomorphic deformations of special geometry and their applications, Springer Proc. Phys. 144 (2013) 1 [arXiv:1206.0577] [INSPIRE].

[49] G. Lopes Cardoso and A. Veliz-Osorio, On the $\sigma$-model of deformed special geometry, Nucl. Phys. B $\mathbf{8 7 2}$ (2013) 228 [arXiv:1212.4364] [INSPIRE].

[50] G.L. Cardoso, B. de Wit and S. Mahapatra, Deformations of special geometry: in search of the topological string, JHEP 09 (2014) 096 [arXiv:1406.5478] [INSPIRE].

[51] K. Behrndt et al., Classical and quantum $N=2$ supersymmetric black holes, Nucl. Phys. B 488 (1997) 236 [hep-th/9610105] [INSPIRE].

[52] G. Lopes Cardoso, B. de Wit and T. Mohaupt, Corrections to macroscopic supersymmetric black hole entropy, Phys. Lett. B 451 (1999) 309 [hep-th/9812082] [INSPIRE].

[53] G. Lopes Cardoso, B. de Wit, J. Kappeli and T. Mohaupt, Stationary BPS solutions in $N=2$ supergravity with $R^{2}$ interactions, JHEP 12 (2000) 019 [hep-th/0009234] [INSPIRE].

[54] S. Ferrara and O. Macia, Real symplectic formulation of local special geometry, Phys. Lett. B 637 (2006) 102 [hep-th/0603111] [INSPIRE].

[55] D.V. Alekseevsky and V. Cortés, Geometric construction of the r-map: from affine special real to special Kähler manifolds, Comm. Math. Phys. 291 (2009) 579 [arXiv:0811.1658] [INSPIRE].

[56] P. Galli, T. Ortín, J. Perz and C.S. Shahbazi, Non-extremal black holes of $N=2, D=4$ supergravity, JHEP 07 (2011) 041 [arXiv: 1105.3311] [INSPIRE].

[57] P. Dempster, Time-like reductions of supergravity and black string solutions, Ph.D. Thesis, University of Liverpool, Liverpool, U.K. (2014). 
[58] G.W. Gibbons, Supersymmetric soliton states in extended supergravity theories, [INSPIRE].

[59] G.W. Gibbons and C.M. Hull, A Bogomolny Bound for General Relativity and Solitons in $N=2$ Supergravity, Phys. Lett. B 109 (1982) 190 [InSPIRE].

[60] S. Ferrara, R. Kallosh and A. Strominger, $N=2$ extremal black holes, Phys. Rev. D 52 (1995) 5412 [hep-th/9508072] [INSPIRE].

[61] G. Bossard and S. Katmadas, Duality covariant multi-centre black hole systems, JHEP 08 (2013) 007 [arXiv: 1304.6582] [INSPIRE].

[62] P. Bueno, P. Galli, P. Meessen and T. Ortín, Black holes and equivariant charge vectors in $N=2, d=4$ supergravity, JHEP 09 (2013) 010 [arXiv: 1305.5488] [INSPIRE].

[63] D.D.K. Chow and G. Compère, Seed for general rotating non-extremal black holes of $\mathcal{N}=8$ supergravity, Class. Quant. Grav. 31 (2014) 022001 [arXiv:1310.1925] [INSPIRE].

[64] P. Dempster, D. Errington and T. Mohaupt, From submanifolds to non-extremal solutions, work in progress.

[65] S. Hawking and G. Ellis, The large scale structure of space-time, Cambridge University Press, Cambridge, U.K. (1973).

[66] R.M. Wald, General Relativity, The University of Chicago Press, Chicago, U.S.A. (1984).

[67] B. Schmidt, Isometry groups with surface-orthogonal trajectories, Z. Naturforschung A 22 (1967) 1351. 